\title{
Feix-Kaledin metric on the total spaces of cotangent bundles to Kähler quotients
}

\author{
Anna Abasheva
}

\begin{abstract}
In this paper we study the geometry of the total space $Y$ of a cotangent bundle to a Kähler manifold $N$ where $N$ is obtained as a Kähler reduction from $\mathbb{C}^{n}$. Using the hyperkähler reduction we construct a hyperkähler metric on $Y$ and prove that it coincides with the canonical Feix-Kaledin metric. This metric is in general non-complete. We show that the metric completion $\widetilde{Y}$ of the space $Y$ is equipped with a structure of a stratified hyperkähler space (in the sense of [31]). We give a necessary condition for the Feix-Kaledin metric to be complete using an observation of R. Bielawski. Pick a complex structure $J$ on $\widetilde{Y}$ induced from the quaternions. Suppose that $J \neq \pm I$ where $I$ is the complex structure whose restriction to $Y=T^{*} N$ is induced by the complex structure on $N$. We prove that the space $\widetilde{Y}_{J}$ admits an algebraic structure and is an affine variety.
\end{abstract}

\section{Contents}

1 Introduction 1

2 Preliminaries on Quotients 4

2.1 Kähler and hyperkähler reduction . . . . . . . . . . . . . 4

2.2 Singular quotients . . . . . . . . . . . . . . . . 6

2.3 Algebraic and analytic quotients . . . . . . . . . . . . 7

2.4 Kähler quotients as good quotients . . . . . . . . . . . . . 11

3 Geometry of Total Spaces of Cotangent Bundles 15

3.1 Semistable points on cotangent bundles . . . . . . . . . . . . 15

3.2 Quotients of affine spaces . . . . . . . . . . . . . 16

3.3 Metric completions of cotangent bundles . . . . . . . . . . . . . 20

3.4 Example: Hirzebruch surfaces . . . . . . . . . . . . . . . 20

4 Cotangent Bundles as Stein and Affine Varieties 23

4.1 Twisted complex structure on cotangent bundle . . . . . . . . . . 23

4.2 Stein structure . . . . . . . . . . . . . . . . . . 26

4.3 Big and nef tangent bundles . . . . . . . . . . . . . . 28

5 Appendix: Feix Construction and Twisted Cotangent Bundles 32

\section{Introduction}

Many known examples of non-compact hyperkähler manifolds arise as total spaces of cotangent bundles to certain Kähler manifolds or contain them as 
an open dense subset ([29], [5], [4], [35] ). Moreover, all these examples are equipped with a natural $U(1)$-action compatible with the hyperkähler structure in the sense of the following definition.

Definition 1.1: Let $X$ be a hyperkähler manifold with the hyperkähler metric $g$ and the holomorphic symplectic form $\Omega \in \Lambda^{2,0} M$. A holomorphic $U(1)$-action on $X$ is said to be HKLR-compatible with the hyperkähler structure if:

(i) The metric $g$ is $U(1)$-invariant.

(ii) $\mathrm{L}_{\varphi} \Omega=\sqrt{-1} \Omega$ where $\varphi$ is the vector field tangent to the action (equivalently, $\lambda^{*} \Omega=\lambda \Omega$ for every $\left.\lambda \in U(1)\right)$.

(iii) The fixed-point set of the $U(1)$-action is a complex Lagrangian submanifold.

Remark 1.2: The $U(1)$-action defined above does not preserve all the complex structures but rotates them. Circle actions preserving all the complex structures will not be considered in this paper. However, they arise in the context of hK/qK-correspondence ([19]) which links both types of circle actions with quaternionic Kähler geometry.

A universal way to construct hyperkähler manifolds with a HKLR-compatible $U(1)$-action is provided by the following theorem proved independently by $\mathrm{D}$. Kaledin and B. Feix.

Theorem 1.3: ([11],[12],[27],[28]) Let $M$ be a Kähler manifold such that the corresponding Kähler metric is real analytic. Then there exists a $U(1)$-invariant neighbourhood $X$ of the zero section of $T^{*} M$ which admits a hyperkähler structure (Definition 2.4) $(g, I, J, K)$ satisfying the following properties:

(i) Consider the natural complex structure $I$ on $T^{*} M$ induced by the complex structure on $M$. Then the holomorphic symplectic form $\Omega \in \Lambda_{I}^{2,0} X$ induced by the hyperkähler structure is the restriction to $X$ of the canonical holomorphic symplectic form on $T^{*} M$ (formula (2.10)).

(ii) The natural $U(1)$-action on $X$ by fiberwise rotations is HKLR-compatible with the hyperkähler structure.

(iii) The Kähler structure $(g, I)$ restricts to the given Kähler structure on $M$.

Moreover this hyperkähler structure is unique up to a linear automorphism of $X$.

Definition 1.4: We shall call the hyperkähler structure on $X$ uniquely defined by Theorem 1.3 the Feix-Kaledin hyperkähler structure. 
Remark 1.5: A nice construction of the Feix-Kaledin hyperkähler structure in a more general setting was given by A. Borówka and D. Calderbank in [6]. Their method is close to the one of B. Feix. A simpler and more explicit geometric approach was discovered by R. Bielawski in [3]. However, his methods are limited to a certain class of Kähler manifolds, namely complexifications of Riemannian manifolds.

Our goal in this paper is to study a series of examples of Kähler manifolds $M$ such that $T^{*} M$ admits globally defined Feix-Kaledin metric. These examples are obtained via Kähler reduction from finite dimensional unitary representations. We shall postpone the description of their construction to the next section and now formulate the main theorem.

Theorem 1.6: Let $V$ be a unitary representation of a compact group $C$ and $T^{*} V$ the total space of its cotangent bundle with the natural hyperkähler metric. Denote by $N:=V / / C$ the Kähler quotient of $V$ by the Hamiltonian action of $C$. The following assertions hold:

(i) The Feix-Kaledin hyperkäler metric on $T^{*} N$ is defined globally.

(ii) There exists a natural open embedding of the hyperkähler manifold $T^{*} N$ to the hyperkähler quotient $\left(T^{*} V\right) / / / C$, moreover, the last variety is complete as a metric space.

We are able to give a necessary condition for the Feix-Kaledin metric on $T^{*} N$ to be complete.

Theorem 1.7: Let $N=V / / C$ be a Kähler quotient of a vector space $V$. Suppose that $N$ is smooth and compact. If the Feix-Kaledin metric on $Y=T^{*} N$ is complete then the tangent bundle $T N$ to the complex manifold $N$ is big and nef.

All the fibers of the twistor space of $\left(T^{*} V\right) / / / C$ except for those over $\pm I$ are isomorphic. This is true for every complete hyperkähler manifold equipped with a HKLR-compatible $U(1)$-action ([21]). In addition, in our case all the fibers are algebraic.

Theorem 1.8: Consider $\left(T^{*} V\right) / / / C$ as a complex analytic variety equipped with some complex structure $J \in \mathbb{H}$. Assume that $J \neq \pm I$. Then $\left(T^{*} V\right) / / / C$ admits a structure of an affine algebraic variety. Moreover, with these assumptions on $J$ the isomorphism class of $\left(\left(T^{*} V\right) / / / C\right)_{J}$ does not depend on the choice of $J$.

The paper is organised as follows. In Section 2 we review the general properties of Kähler, hyperkähler and good quotients and relations between them. 
The only results in this section which are specifically relevant to our situation are Proposition 2.23, Proposition 2.24 and Corollary 2.39. They seem to be not explicitly written anywhere. In Section 3 we give a precise formulation of Theorem 1.6 and prove it. Section 4 deals with the proof of Theorem 1.8. We also study the moment map $\psi:\left(T^{*} V\right) / / / C \rightarrow \mathbb{R}_{\geq 0}$ for the $U(1)$-action. We prove that the function $-\psi$ is proper and strictly plurisubharmonic. Thus, we obtain a weaker statement that this hyperkähler quotient is a Stein variety ([13]). Further, we show that if the Feix-Kaledin metric is complete then the tangent bundle of $V / / C$ is big and nef. We do this using our previous result on the properness of $\psi$ and an unpublished result due to R.Bielawski. In Appendix we rewrite the Feix construction of the Feix-Kaledin hyperkähler metric in a more canonical language. That allows us to see its relations to twisted cotangent bundles.

Acknowledgements: I would like to thank my adviser Misha Verbitsky for suggesting me the problem. I am grateful to Dmitry Kaledin, Roger Bielawski and anonymous referees for reading a draft of this paper and their remarks on its content. I also thank Enrico Arbarello and Ignasi Mundet i Riera for useful remarks on certain parts of the proof. My thanks also go to my friend Renat Abugaliev for his interest and encouraging discussions. I was partially supported by the HSE University Basic Research Program, Russian Academic Excellence Project '5-100' as well as by Independent University of Moscow and Instituto de Matemática Pura e Aplicada.

\section{Preliminaries on Quotients}

\subsection{Kähler and hyperkähler reduction}

Let $X$ be a Kähler manifold, $\omega \in \Lambda^{1,1}(X)$ a Kähler form and $C$ a compact group acting on $X$. Suppose that the action of $C$ preserves the Kähler structure. Denote the Lie algebra of $C$ by $\mathfrak{c}$. The differential of the $C$-action on $X$ induces a morphism of Lie algebras $L: \mathfrak{c} \rightarrow \Gamma(T X)$ defined as usual by the formula

$$
L(\xi)_{x}=\left.\frac{d}{d t} \exp (t \xi) x\right|_{t=0}
$$

for every $x \in M$. For convenience we shall slightly abuse the notation by denoting a vector $\xi \in \mathfrak{c}$ and its image $L(\xi)$ in vector fields on $X$ by the same symbol $\xi$.

Definition 2.1: The action of $C$ on a Kähler manifold $X$ is called Hamiltonian if there exists a map $\mu: X \rightarrow \mathfrak{c}^{*}$ satisfying the following properties:

(i) For every $\xi \in \mathfrak{c}$

$$
d\langle\mu, \xi\rangle=\xi\lrcorner \omega \in \Omega^{1}(X, \mathbb{R})
$$


(ii) (C-equivariance) For every $g \in C$

$$
\mu(g x)=A d^{*} g \cdot \mu(x)
$$

If $C$ is connected this is equivalent to saying that for every $\xi, \eta \in \mathfrak{c}$

$$
\omega(\xi, \eta)=-\langle\mu,[\xi, \eta]\rangle
$$

In that case $\mu$ is called a moment map.

Remark 2.2: The definition does not make use of the complex structure on $X$ and can be given for every symplectic manifold $X$ on which a Lie group $C$ acts by symplectomorphisms. However, we shall restrict our attention only to the Kähler and later to the hyperkähler case.

Remark 2.3: A moment map is defined uniquely up to an addition of an $A d^{*}$ invariant element of $\mathfrak{c}^{*}$.

One can introduce a similar notion in the case when $X$ is a hyperkähler manifold and $C$ acts by preserving the hyperkähler structure. We recall here the definition of a hyperkähler manifold.

Definition 2.4: Suppose $(X, g)$ is a Riemannian manifold equipped with an algebra map $\mathbb{H} \rightarrow \operatorname{End}(T X)$ where $\mathbb{H}$ is the quaternion algebra. Suppose also that every complex structure $J \in \mathbb{H} \subset \operatorname{End}(T X)$ is integrable and orthogonal with respect to the metric $g$. For every complex structure $J \in \mathbb{H}$ define

$$
\omega_{J}(v, u):=g(J v, u)
$$

If $\omega_{J}$ is closed for every $J$ then the manifold $X$ is called hyperkähler.

For every hyperkähler manifold $X$ we fix a triple of complex structures $(I, J, K) \in \operatorname{End}(T X)$ satisfying quaternionic relations $I J=-J I=K$.

Definition 2.5: Let $C$ be a compact connected Lie group acting on a hyperkähler manifold $X$. Suppose that the action of $C$ preserves the hyperkähler structure. The action is called Hamiltonian if there exists a map $\mu: X \rightarrow \mathfrak{c}^{*} \otimes \mathbb{R}^{3}$

$$
\mu=\left(\mu_{I}, \mu_{J}, \mu_{K}\right)
$$

such that $\mu_{I}, \mu_{J}$ and $\mu_{K}$ are moment maps with respect to Kähler forms $\omega_{I}, \omega_{J}$ and $\omega_{K}$ respectively (in the sense of Definition 2.1). The map $\mu: X \rightarrow \mathfrak{c}^{*} \otimes \mathbb{R}^{3}$ is called a hyperkähler moment map.

Theorem 2.6: ([21]) Let $X$ be a Kähler (resp. hyperkähler) manifold equipped with a Hamiltonian action of a compact Lie group $C$ with a moment map $\mu: X \rightarrow \mathfrak{c}^{*}\left(\right.$ resp. $\left.\mu: X \rightarrow \mathfrak{c}^{*} \otimes \mathbb{R}^{3}\right)$. Consider the subspace $Z:=\mu^{-1}(0) \subset X$. Then the following holds 
(i) The subspace $Z$ is $C$-invariant.

(ii) Suppose $C$ acts on $Z$ freely. Then zero is a regular value of $\mu$, hence $Z$ is a submanifold of $X$. The quotient $Y:=\mu^{-1}(0) / C$ is equipped with a natural structure of a Kähler (resp. hyperkähler) manifold. This Kähler (resp. hyperkähler) structure is the unique one such that for every $x \in Z$ projecting to a point $y \in Y$ the natural projection map from $\operatorname{im}(\mathfrak{c})^{\perp} \subset T_{x} Z$ to $T_{y} Y$ is an isomorphism of Hermitian (resp. hyper-Hermitian) vector spaces.

Definition 2.7: The manifold $Y$ from Theorem 2.6 is called a Kähler (resp. hyperkähler) quotient of $X$. In the Kähler case it is denoted by $X / / C$ and in the hyperkähler case by $X / / / C$.

\subsection{Singular quotients}

In this subsection we state some more or less technical definitions and theorems to be used later. The reader is advised to skip it until needed.

Let $C$ be a compact Lie group acting on a manifold $X$ and $D \subset C$ be its subgroup. For a point $x \in X$ let $\operatorname{Stab}(x)$ be the stabilizer of $x$ in $C$. Following [38] we introduce the following notation

$$
\begin{gathered}
X^{D}:=\{x \in X \mid D \subset \operatorname{Stab}(x)\} \\
X_{D}:=\{x \in X \mid D=\operatorname{Stab}(x)\} \\
X_{(D)}:=\{x \in X \mid \operatorname{Stab}(x) \text { is conjugate to } D\}
\end{gathered}
$$

As $C$ is compact the subset $X^{D}$ is a submanifold of $X$ containing $X_{D}$ as a dense open subset. Consider the normalizer $N(D)$ of the subgroup $D$ in $C$ and denote $W(D):=N(D) / D$. Then the group $W(D)$ acts properly and freely on $X_{D}$ and $X_{(D)} / C=X_{D} / W(D)$ is a disjoint union of smooth submanifolds.

Now let us return to the case when $X$ is Kähler and the action of $C$ is Hamiltonian with a moment map $\mu: X \rightarrow \mathfrak{c}^{*}$.

Theorem 2.8: ([38]) The subsets $X_{D}$ are locally closed Kähler submanifolds of $M$. Moreover, the restriction of the moment map $\mu: X \rightarrow \mathfrak{c}^{*}$ to $X_{D}$ induces the moment map $\mu_{D}: X_{D} \rightarrow \mathfrak{w}_{D}^{*}$ where $\mathfrak{w}_{D}$ is the Lie algebra of $W(D)$. Zero is a regular value of $\mu_{D}$ and $W(D)$ acts freely on $X_{D} \cap Z$. As a result the quotient $Y$ of $Z=\mu^{-1}(0)$ by $C$ decomposes as a union of Kähler manifolds $Y_{(D)}:=\left(X_{(D)} \cap Z\right) / C=\left(X_{D} \cap Z\right) / W(D)$.

Remark 2.9: In fact Sjamaar and Lerman in [38] prove a stronger statement, in particular, they show that the decomposition $Y=\bigcup Y_{(D)}$ is a stratification.

A similar theorem holds in the hyperkähler case. 
Theorem 2.10: ([26],[9]) Let $X$ be a hyperkähler manifold with a Hamiltonian action of a group $C$. Let $\mu: X \rightarrow \mathfrak{c}^{*} \otimes \mathbb{R}^{3}$ be a moment map. Then the hyperkähler quotient $Y=X / / C$ decomposes as a union of hyperkähler manifolds $Y_{(D)}:=\left(X_{(D)} \cap Z\right) / C$.

Remark 2.11: The decomposition of the hyperkähler quotient $X / / C$ as a union of hyperkähler manifolds endows it with a structure of a stratified hyperkähler space. We refer to [31] for definitions and proofs.

\subsection{Algebraic and analytic quotients}

The procedure of taking quotients by non compact groups may sometimes be a subtle question. Naïve topological quotients might look too ugly to work with. There exists a definition of a good quotient which mimics some standard properties of topological quotients and works well in the category of algebraic varieties over $\mathbb{C}$ or the category of complex analytic varieties. Either of the two mentioned categories will be denoted by $C$.

Definition 2.12: ([23], Section 3.5; [15], Section $2.2 \&$ 2.3) Let $X$ be an object of $C$ equipped with an action of a complex group $G \in O b(C)$. We call a $G$-invariant surjective map $p: X \rightarrow Y$ a good quotient map if it satisfies the following properties:

(i) The map $p$ is affine (resp. locally Stein) i.e. the preimage of every affine (resp. Stein) open subset of $Y$ is affine (resp. Stein).

(ii) The natural map $\mathcal{O}_{Y} \rightarrow\left(p_{*} \mathcal{O}_{X}\right)^{G}$ is an isomorphism of sheaves.

(iii) $p$ maps $G$-invariant closed subvarieties to closed subvarieties.

(iv) $p$ maps disjoint $G$-invariant closed subvarieties to disjoint subsets.

Remark 2.13: Good quotients are often called Hilbert quotients especially in the analytic context.

Remark 2.14: Good quotients are always categorical quotients in the following sense. Every $G$-invariant map from $X$ to some $Y^{\prime} \in O b(C)$ can be uniquely factorized through the projection $p$ from $X$ to the good quotient $Y$ ([23], Prop. $3.30)$.

We shall denote the good quotient of $X$ by $G$ by the symbol $X / G$. This is not the notation commonly used in the literature but it seems that it will enable us to avoid confusion with Kähler quotients.

The only thing we shall need to know about the existence of good quotients is the following. 
Proposition 2.15: ([32], Ch. 1, §2, Thm. 1.1; [23], Thm. 4.30; [16]) Let $X \in O b(C)$ be a complex variety equipped with an action of a complex reductive group $G$.

(i) Suppose that $X$ is affine (resp. Stein). Then $X$ admits a good quotient by $G$.

(ii) Suppose that $X$ admits an affine (resp. locally Stein) $G$-equivariant map to a $G$-variety $M$. Suppose that $M$ admits a good quotient $N$. Then $X$ admits a good quotient $Y$ and $Y$ is affine (resp. locally Stein) over $N$.

Remark 2.16: The proof in the algebraic case would follow from the following fact. If $X=\operatorname{Spec}(A)$ where $A$ a $\mathbb{C}$-algebra of finite type then the good quotient $X / G$ is isomorphic to $\operatorname{Spec}\left(A^{G}\right)$. The second assertion follows from the first as good quotients are always affine.

The next theorem relates quotients in algebraic and analytic categories.

Theorem 2.17: ([30],[20]) Let $X$ be an algebraic variety over $\mathbb{C}$ and $G$ a reductive group acting on $X$ algebraically. Assume that there exists a good quotient map $p: X \rightarrow Y$ to an algebraic variety $Y$. Then $p: X^{a n} \rightarrow Y^{a n}$ is a good quotient map in the category of complex analytic varieties.

Definition 2.18: A good quotient $p: X \rightarrow X / G=: Y$ is called a geometric quotient if the preimage of any point of $Y$ is a closed orbit in $X$.

Equivalently, a good quotient is geometric if every $G$-orbit in $X$ is closed ([23], Cor. 3.32). Though not all good quotients are geometric, there is an important case when they are.

Proposition 2.19: Let $G$ be a reductive group acting on $X$ with finite stabilizers. If $X$ admits a good quotient by $G$ then this quotient is geometric. Proof: The assumption on stabilizers guarantees that all orbits have the same dimension equal to $\operatorname{dim} G$. If there was a non-closed orbit $G \cdot x$ then its closure $\overline{G \cdot x}$ would contain an orbit of smaller dimension.

We finish the subsection with an example of a quotient which will be pivotal in the sequel. The motivation for this example comes from the procedure of a holomorphic symplectic reduction which we shall now describe. Let $X$ be a holomorphic symplectic variety with a holomorphic symplectic form $\Omega \in \Lambda^{2,0} X$. Suppose $X$ is equipped with an action of a complex reductive group $G$ preserving holomorphic symplectic structure. The definition below mimics Definition 2.1.

Definition 2.20: The action of $G$ on a holomorphic symplectic variety $X$ is called Hamiltonian if there exists a map $m: X \rightarrow \mathfrak{g}^{*}$ with the following 
properties:

(i) For every $\xi \in \mathfrak{g}$

$$
d\langle m, \xi\rangle=\xi\lrcorner \Omega \in \Lambda^{1,0}(X)
$$

(ii) (G-equivariance) For every $g \in G$

$$
m(g x)=A d^{*} g \cdot m(x)
$$

The map $m: X \rightarrow \mathfrak{g}^{*}$ is called a holomorphic symplectic moment map.

Remark 2.21: The moment map $m$ is automatically holomorphic.

Similarly to Theorem 2.6 one can consider a $G$-invariant subvariety $Z:=$ $m^{-1}(0) \subset X$. If $Z$ admits a good quotient $Y$ then $Y$ inherits the holomorphic symplectic structure from $X$.

Definition 2.22: Suppose $Z$ admits a good quotient. Then we call $Z / G$ a holomorphic symplectic quotient of $X$ and denote it by $X / / G$.

Now let $M \in O b(\mathrm{C})$ be a smooth variety and $X$ the total space of its cotangent bundle. Let $G \in O b(C)$ be a complex reductive group acting on $M$. The action of $G$ can be naturally extended to $X$ as

$$
g \cdot(x, \alpha):=\left(g x,\left(g^{-1}\right)^{*} \alpha\right)
$$

where $x \in M, \alpha \in T_{x}^{*} M$. The inclusion $\iota$ of $M$ as the zero section and the projection $\pi$ from $X$ to $M$ become $G$-equivariant maps with respect to this action.

The manifold $X$ is equipped with the standard holomorphic symplectic 2form $\Omega \in \Lambda^{2,0}(X)$. More precisely,

$$
\Omega=-d \tau
$$

where $\tau$ is the tautological holomorphic 1-form on $X$. Recall that $\tau$ is defined as follows

$$
\tau_{(x, \alpha)}(v):=\alpha\left(\pi_{*} v\right)
$$

for every $v \in T_{(x, \alpha)} X$.

Proposition 2.23: The action of $G$ on $X$ preserves $\tau$ and hence $\Omega$. It is Hamiltonian with a moment map $m: X \rightarrow \mathfrak{g}^{*}$ (Definition 2.20)

$$
\langle m(x, \alpha), \xi\rangle=\langle\alpha, \xi\rangle
$$

where on the right-hand side we consider $\xi$ to be an element of $T_{x} M$.

Proof: By definition

$$
\tau_{(x, \alpha)}(v)=\alpha\left(\pi_{*} v\right)
$$


Hence for every $g \in G$

$$
\begin{gathered}
g^{*} \tau_{(x, \alpha)}(v)=\tau_{\left(g x,\left(g^{-1}\right)^{*} \alpha\right)}\left(g_{*} v\right)= \\
=\left(g^{-1}\right)^{*} \alpha\left(\pi_{*} g_{*} v\right)=\left(g^{-1}\right)^{*} \alpha\left(g_{*} \pi_{*} v\right)= \\
=\alpha\left(\pi_{*} v\right)=\tau_{(x, \alpha)}(v)
\end{gathered}
$$

and the $G$-action preserves $\tau$. We obtain that for every $\xi \in \mathfrak{g}$

$$
\left.\left.0=\mathrm{L}_{\xi} \tau=d(\xi\lrcorner \tau\right)-\xi\right\lrcorner \Omega
$$

and

$$
\langle m(x, \alpha), \xi\rangle:=\xi\lrcorner \tau=\langle\alpha, \xi\rangle
$$

is a moment map. The $G$-equivariance of $m$ is easily checked.

Proposition 2.24: Suppose that $G$ acts on a smooth variety $M$ with trivial stabilizers and $M$ admits a good (and therefore geometric) quotient $p: M \rightarrow$ $M / G=: N$. Then:

(i) The variety $N$ is smooth.

(ii) The subvariety $Z:=m^{-1}(0) \subset X:=T^{*} M$ is smooth and admits a smooth geometric quotient $Y:=Z / G$.

(iii) The quotient $Y$ is naturally isomorphic to $T^{*} N$.

\section{Proof:}

(i) Let $x$ be a point of $M$. Choose any smooth complex analytic subvariety $U$ in a neighbourhood of $x$, transversal to $G \cdot x$ and having complementary dimension to this orbit. By choosing $U$ sufficiently small we can guarantee that $U$ is mapped isomorphically onto a neighbourhood of $p(x)$.

(ii) For smoothness of $m^{-1}(0)$ it is enough to prove that the differential $d m: T_{x} X \rightarrow \mathfrak{g}^{*}$ is surjective for any point $x \in m^{-1}(0)$. We describe the kernel of $d m$ to show this.

$$
\begin{gathered}
\left.\operatorname{ker} d m=\left\{v \in T_{x} X \mid v\right\lrcorner d\langle m, \xi\rangle=0 \forall \xi \in \mathfrak{g}\right\}= \\
=\left\{v \in T_{x} X \mid \Omega(\xi, v)=0 \forall \xi \in \mathfrak{g}\right\}=\left(\operatorname{im}\left(\mathfrak{g} \rightarrow T_{x} X\right)\right)^{\perp_{\Omega}}
\end{gathered}
$$

As $G$ acts on $M$ with trivial stabilizers, it also must act with trivial stabilizers on $X$. Hence the map $\mathfrak{g} \rightarrow T_{x} X$ is injective for any $x \in X$. The dimension count shows $\operatorname{dim}_{\mathbb{C}}(\operatorname{ker} d M)=\operatorname{dim}_{\mathbb{C}} X-\operatorname{dim}_{\mathbb{C}} G$. The differential $d M$ is therefore surjective.

The projection map $\pi: Z \rightarrow M$ is clearly affine (if $M$ is assumed to be an algebraic variety) or locally Stein (if $M$ is assumed to be a complex analytic variety). Proposition 2.15 guarantees that $Z$ admits a good quotient $Y:=$ $Z / G$ as soon as $M$ does. By Proposition 2.19 the quotient $Y$ is geometric. The proof of the first part of the proposition applied to the quotient $Y=$ $Z / G$ shows that $Y$ is smooth. 
(iii) The map $f: T^{*} N \rightarrow Y$ is defined as follows. Let $y$ be a point of $N$. Choose any $x \in p^{-1}(y) \subset M$. The pullback map $p^{*}: T_{y}^{*} N \rightarrow T_{x}^{*} M$ maps $T_{y}^{*} N$ isomorphically onto the space of elements of $T_{x}^{*} M$ vanishing on the tangent space in $x$ to the orbit $G \cdot x$. Proposition 2.23 implies that for every $\alpha \in T_{y}^{*} N$ the value of $m$ at $p^{*} \alpha$ vanishes. We define $f(\alpha) \in Y=m^{-1}(0) / G$ to be the image of $p^{*} \alpha \in m^{-1}(0)$ in $Y$. One can check that the map is defined correctly and is an isomorphism.

The following proposition is a classical fact about submersions. We shall omit its proof.

Proposition 2.25: Suppose that $G$ acts on a smooth variety $M$ with trivial stabilizers and $p: M \rightarrow N$ is the quotient map. For every $r \in \mathbb{Z}_{>0}$ let $\Omega_{h o r}^{r} \subset$ $\Omega^{r} M$ be the subsheaf of horizontal $r$-forms i.e. those $r$-forms $\alpha \in \Omega^{r} M$ such that $\xi\lrcorner \alpha=0$ for every $\xi \in \mathfrak{g}$. Then the pullback map on $r$-forms

$$
\Omega^{r} N \rightarrow\left(p_{*} \Omega_{h o r}^{r}\right)^{G}
$$

and the pushforward map on vector fields

$$
\left(p_{*}\left(\mathcal{T} M / \mathfrak{g} \cdot \mathcal{O}_{M}\right)\right)^{G} \rightarrow \mathcal{T} N
$$

are both isomorphisms of sheaves of $\mathcal{O}_{N}$-modules.

\subsection{Kähler quotients as good quotients}

Our goal now is to present a Kähler quotient of a Kähler manifold $X$ as a geometric or at least good quotient of some open subset of $X$. An interested reader can consult a survey [14] for the compact case and a survey [20] for the non-compact case.

Definition 2.26: Let $G$ be a complex reductive group acting holomorphically on a Kähler manifold $X$. Suppose that the restriction of this action to a fixed maximal compact subgroup $C$ of $G$ preserves the Kähler structure and is Hamiltonian with a moment map $\mu: X \rightarrow \mathfrak{c}^{*}$.

(i) A point $x \in X$ is called semistable if

$$
\overline{G x} \cap \mu^{-1}(0) \neq \varnothing
$$

We denote the set of semistable points of $X$ by $X^{s s}$. We shall call a point unstable if it is not semistable. We shall denote the set of unstable points by $X^{u s}$. 
(ii) A point $x \in X$ is called polystable if

$$
G x \cap \mu^{-1}(0) \neq \varnothing
$$

We denote the set of polystable points as $X^{p s}$.

(iii) A point $x \in X$ is called stable if it is polystable and the stabilizer of $x$ in $G$ is finite. We denote the set of stable points as $X^{s}$

It follows easily from the definitions that the sets $X^{s s}, X^{u s}, X^{p s}$ and $X^{s}$ are $G$-invariant.

Proposition 2.27: ([20],[14], Thm. 7.2) The subsets $X^{s}$ and $X^{s s}$ are open in $X$.

Remark 2.28: The set $X^{p s}$ of polystable points is not necessarily open.

Now we want to construct a map from $X^{s s}$ to $\mu^{-1}(0) / C$ i.e. to the Kähler quotient of $X$. To do this we need the following two results.

Lemma 2.29: Suppose $x, y \in \mu^{-1}(0)$ lie in the same $G$-orbit. Then they lie in the same $C$-orbit.

Proof: Let $y=g x$. By the polar decomposition $g=g_{0} \exp (\sqrt{-1} \xi)$ for some $g_{0} \in C$ and $\xi \in \mathfrak{c}$. Consider the curve $y(t)=\exp (\sqrt{-1} t \xi) g_{0} x$ connecting $g_{0} x$ and $y$, then

$$
\left.\frac{d}{d t}\left\langle\mu\left(y_{t}\right), \xi\right\rangle\right|_{t=\tau}=\omega\left(\xi_{y(\tau)}, I \xi_{y(\tau)}\right)=\left\|\xi_{y(\tau)}\right\|^{2} \geq 0
$$

As $\mu\left(g_{0} x\right)=\mu(y)=0$ we see that $\xi_{g_{0} x}=0$ and hence $y=g_{0} x$.

Lemma 2.30: ([20], Cor. 4.2.2) Every semistable orbit contains a unique polystable orbit in its closure.

Now we can define a map $\Psi: X^{s s} \rightarrow \mu^{-1}(0) / C$ as follows. For a semistable point $x \in X$ consider the unique polystable orbit in the closure of its $G$-orbit. It intersects $\mu^{-1}(0)$ in a $C$-orbit $\widetilde{x} \in \mu^{-1}(0) / C$. We define

$$
\Psi(x):=\widetilde{x}
$$

Proposition 2.31: ([20], Thm. 4.2.4) Let $G$ be a complex reductive group acting holomorphically on a Kähler manifold $X$. Suppose that the restriction of this action to a fixed maximal compact subgroup $C$ of $G$ preserves the Kähler structure and is Hamiltonian with a moment map $\mu: X \rightarrow \mathfrak{c}^{*}$.

(i) The map $\Psi: X^{s s} \rightarrow \mu^{-1}(0) / C$ is a good quotient map. 
(ii) Let $\mu^{-1}(0)^{s}$ be the set of points in $\mu^{-1}(0)$ whose stabilizer in $C$ is finite. Then the map $\left.\Psi\right|_{X^{s}}: X^{s} \rightarrow \mu^{-1}(0)^{s} / C$ is a geometric quotient.

Corollary 2.32: Assume that zero is a regular value of the moment map. Then all the inclusions $X^{s} \subset X^{p s} \subset X^{s s}$ are equalities. Moreover, the map $\Psi: X^{s} \rightarrow \mu^{-1}(0) / C$ is a geometric quotient.

Proof: If $x \in X$ be a polystable point, then the orbit $G \cdot x$ intersects $\mu^{-1}(0)$. Every point of $\mu^{-1}(0)$ has finite stabilizer. Hence the stabilizer of $x$ is also finite and $x$ is stable. If $x \in X$ is semistable but not polystable then $\overline{G \cdot x}$ contains a polystable orbit $G \cdot y \operatorname{such}$ that $\operatorname{dim} G \cdot y<\operatorname{dim} G \cdot x$. But as we have seen earlier the point $y$ must have finite stabilizer, hence $\operatorname{dim} G \cdot y$ is maximal, contradiction.

It is usually non-trivial to say if a given point is (semi)stable or not. One of the ways which can be practically used to answer this question is the HilbertMumford criterion which we shall state after giving the necessary definition.

Definition 2.33: The $\mu$-weight of a pair $(x, \xi) \in X \times \mathfrak{c}$ is a number $w_{\mu}^{\xi}(x) \in$ $\mathbb{R} \cup\{ \pm \infty\}$ defined by the equation

$$
w_{\mu}^{\xi}(x):=\lim _{t \rightarrow \infty}\langle\mu(\exp (\sqrt{-1} t \xi) x), \xi\rangle
$$

Remark 2.34: One can prove that the limit in formula (2.22) is always welldefined. Indeed, the function $\psi(t):=\langle\mu(\exp (\sqrt{-1} t \xi) x), \xi\rangle$ is non-decreasing as

$$
\frac{d}{d t} \psi=\omega(\xi, I \xi)=\|\xi\|^{2} \geq 0
$$

by the very definition of a moment map.

Theorem 2.35: ("Hilbert-Mumford criterion", [33],[39])

(i) A point $x \in X$ is stable if and only if $w_{\mu}^{\xi}(x)>0$ for every $\xi \in \mathfrak{c} \backslash\{0\}$.

(ii) Assume that the action of $G$ on $X$ is energy complete ([39], Def. 2.8). A point $x \in X$ is semistable if and only if $w_{\mu}^{\xi}(x) \geq 0$ for every $\xi \in \mathfrak{c} \backslash\{0\}$.

We shall not need the definition of energy complete actions. By [39], Prop. 2.9 any linear action is energy complete, and this is the only case to which we shall apply the criterion.

Remark 2.36: A similar description of polystable points was given by I. Mundet i Riera in [34].

Let us now apply the Hilbert-Mumford criterion to a specific example. 
Example 2.37: Consider a unitary representation of $C$ in an Hermitian vector space $(V, h)$. The complex manifold $V$ admits the standard Kähler form $\omega=\operatorname{Im} h$ which is preserved by the action of $C$. Moreover the action of $C$ is Hamiltonian and the moment map $\mu: V \rightarrow \mathfrak{c}$ is given by the formula

$$
\langle\mu(v), \xi\rangle=\frac{1}{2} \omega(\xi v, v)+\langle\theta, \xi\rangle
$$

where $\theta$ is some $A d^{*}$-invariant element of $\mathfrak{c}^{*}$. We omit the derivation of this formula.

Let us introduce the notation. Every $\xi \in \mathfrak{c}$ is a skew-symmetric operator on $V$ i.e. $\xi \in \mathfrak{u}(V)$. Hence its eigenvalues are imaginary. We denote them by $\sqrt{-1} \lambda_{i}$. Given a vector $v \in V$ we can write it as the $\operatorname{sum} v=\sum_{i} v_{i}$ where $v_{i}$ lies in the eigenspace corresponding to the eigenvalue $\sqrt{-1} \lambda_{i}$. Consider the set of indices $I_{v}:=\left\{i \mid v_{i} \neq 0\right\}$.

Proposition 2.38: The $\mu$-weight of a pair $(v, \xi)$ is given by the formula

$$
w_{\mu}^{\xi}(v)= \begin{cases}+\infty, & \text { if } \exists i \in I_{v} \text { s.t. } \lambda_{i}<0 \\ \langle\theta, \xi\rangle, & \text { otherwise }\end{cases}
$$

Proof: Let $v=\sum_{i} v_{i}$. Then $\exp (\sqrt{-1} t \xi) v=\sum \exp \left(-\lambda_{i} t\right) v_{i}$. Hence

$$
\begin{gathered}
\left\langle\mu(\exp (\sqrt{-1} t \xi) v, \xi\rangle=\frac{1}{2} \omega\left(I \sum_{i} \lambda_{i} \exp \left(-\lambda_{i} t\right) v_{i}, \sum_{i} v_{i}\right)+\langle\theta, \xi\rangle=\right. \\
=-\frac{1}{2} \sum_{i} \lambda_{i} \exp \left(-\lambda_{i} t\right)\left\|v_{i}\right\|^{2}+\langle\theta, \xi\rangle
\end{gathered}
$$

where the last identity follows from the fact that eigenspaces of a unitary operator are orthogonal. Taking the limit as $t \rightarrow \infty$ we obtain the result.

Corollary 2.39: The set of (semi)stable points of a vector space $V$ is a complement of a union of vector subspaces in $V$, more precisely

$$
V^{s}=V \backslash\left(\bigcup_{\langle\theta, \xi\rangle \leq 0} V^{\xi \geq 0}\right) ; \quad V^{s s}=V \backslash\left(\bigcup_{\langle\theta, \xi\rangle<0} V^{\xi \geq 0}\right)
$$

where the union is taken over $\xi \in \mathfrak{c}$. Here $V^{\xi \geq 0}$ is the sum of eigenspaces of $\xi$ with eigeinvalues $\sqrt{-1} \lambda_{i}$ with non-negative $\lambda_{i}$.

Proof: By Proposition 2.38 the points in $\bigcup_{\langle\theta, \xi\rangle \leq 0} V^{\xi \geq 0}$ are precisely the points with a non-positive $\mu$-weight and the points in $\bigcup_{\langle\theta, \xi\rangle<0} V^{\xi \geq 0}$ are precisely the 
points with a negative $\mu$-weight. By the Hilbert-Mumford criterion (Theorem 2.35) the point is stable if and only if its $\mu$-weight is positive and semistable if and only if its $\mu$-weight is non-negative.

Remark 2.40: It is worth mentioning that an investigation of linear representations from the viewpoint of GIT was carried out by V. Hoskins in [22].

\section{Geometry of Total Spaces of Cotangent Bun- dles}

\subsection{Semistable points on cotangent bundles}

The most basic example of the Feix-Kaledin structure is the standard hyperkähler structure on the total space $X$ of the cotangent bundle of an Hermitian vector space $V$. As a complex vector space $X_{I}=V \oplus V^{*} \cong V \oplus \bar{V}$ where the last isomorphism is induced by the Hermitian metric on $V$. As a real vector space $X_{\mathbb{R}}$ is just the direct sum of two copies of the vector space $V_{\mathbb{R}}$. The complex structures can be written as follows

$$
\begin{gathered}
I(x, y)=(I x,-I y) \\
J(x, y)=(-y, x)
\end{gathered}
$$

and the Riemannian metric is just the direct sum of the metrics on $V$. We slightly abuse the notation by denoting the complex structure $I$ on $V_{\mathbb{R}}$ and on $X_{\mathbb{R}}$ by the same symbol.

We shall now use the arguments and constructions of Example 2.37 to give a description of semistable points on cotangent bundles to Hermitian vector spaces.

Consider a unitary representation of a compact connected Lie group $C$ in an Hermitian vector space $(V, h)$. It can be uniquely extended to the complex representation of the complexification $G$ of $C$. In formula (2.9) we defined an action of the group $G$ on the total space of cotangent bundle to any $G$-manifold. In the same way we define the action of $G$ on the cotangent bundle $X$ to $V$. This action is linear on $X$ as a complex vector space $V \oplus V^{*}$. The representation of $G$ on $X$ is equal to the direct sum of the original representation and its dual. First, we shall consider a more general case of the direct sum of two arbitrary representations.

Lemma 3.1: Let $U=V \oplus W$ be the direct sum of two representations of $C$. Then every moment map $\mu_{V}: V \rightarrow \mathfrak{c}^{*}$ can be extended uniquely to a moment $\operatorname{map} \mu_{U}: U \rightarrow \mathfrak{c}^{*}$.

Proof: Formula (2.23) implies that every moment map $\mu_{U}: U \rightarrow \mathfrak{c}^{*}$ has the form

$$
\left\langle\mu_{U}(x, y), \xi\right\rangle=\frac{1}{2}\left(\omega_{V}(\xi x, x)+\omega_{W}(\xi y, y)\right)+\langle\theta, \xi\rangle
$$


for some $C$-invariant $\theta \in \mathfrak{c}^{*}$. The moment map $\mu_{U}$ is uniquely defined by the covector $\theta \in \mathfrak{c}^{*}$ which can be read from the restriction of $\mu_{U}$ to $V$.

Proposition 3.2: Let $U=V \oplus W$ be the direct sum of two representations of $C$. Denote by $p: U \rightarrow V$ the projection to the first factor. Fix a moment map $\mu_{V}: V \rightarrow \mathfrak{c}^{*}$. Let $\mu_{U}: U \rightarrow \mathfrak{c}^{*}$ be the moment map on $U$ extending $\mu_{V}$. (Its existence and uniqueness is guaranteed by Lemma 3.1.) Let $V^{s}$ (resp. $V^{s s}$ ) be the set of stable (resp. semistable) points of $V$ with respect to the moment map $\mu_{V}$, the analogous notation is used for $U$. Then

$$
p^{-1}\left(V^{s}\right) \subset U^{s} ; \quad p^{-1}\left(V^{s s}\right) \subset U^{s s}
$$

Proof: By applying Corollary 2.39 to $U=V \oplus W$ we obtain that

$U \backslash U^{s}=\bigcup_{\langle\theta, \xi\rangle<0}(V \oplus W)^{\xi \geq 0}=\bigcup_{\langle\theta, \xi\rangle<0}\left(V^{\xi \geq 0} \oplus W^{\xi \geq 0}\right) \subset \bigcup_{\langle\theta, \xi\rangle<0}\left(V^{\xi \geq 0} \oplus W\right)=p^{-1}\left(V \backslash V^{s}\right)$

In other words, $p^{-1}\left(V^{s}\right) \subset U^{s}$. The proof for semistable points is similar.

Corollary 3.3: Let $V$ be a unitary representation of a compact Lie group $C$ and $X$ the cotangent bundle to $V$. Then the preimage of $V^{s}$ (resp. $V^{s s}$ ) under the projection $\pi: X \rightarrow V$ is contained in $X^{s}$ (resp. $X^{s s}$ ).

Proof: Follows by applying Proposition 3.2 to $U=T^{*} V=V \oplus V^{*}$.

\subsection{Quotients of affine spaces}

We consider again an Hermitian vector space $V$ with a unitary linear action of a compact group $C$. We already know that the action of $C$ on $V$ is Hamiltonian i.e. there exists a moment map $\mu: V \rightarrow \mathfrak{c}^{*}$. By Lemma 3.1 this moment map extends to the moment map $\mu_{I}: X \rightarrow \mathfrak{c}^{*}$ where $X:=T^{*} V$. The moment map $\mu_{I}$ and the holomorphic symplectic moment map $m: X \rightarrow \mathfrak{g}^{*}=\mathfrak{c}^{*} \otimes \mathbb{C}$ as in Proposition 2.23 form together a hyperkähler moment map

$$
\mu_{h k}:=\left(\mu_{I}, \operatorname{Re} m, \operatorname{Im} m\right): X \rightarrow \mathfrak{c}^{*} \otimes \mathbb{R}^{3}
$$

Theorem 3.4: Let $V$ be an Hermitian vector space. Suppose that a complex reductive group $G$ acts on $V$ linearly and the restriction of the action of $G$ to some fixed maximal compact subgroup $C \subset G$ preserves the Hermitian structure. Assume that the action of $C$ on $V$ is generically free i.e. the set $V_{0}$ of points with trivial stabilizer is open in $V$. Let $N:=V / / C$ be the Kähler quotient of $V$ by $C$ with respect to a fixed moment map $\mu: V \rightarrow \mathfrak{c}^{*}$. Then the Feix-Kaledin metric is globally defined on the total space $Y_{0}$ of the cotangent bundle to the open Kähler stratum $N_{0}:=V_{0} / / C$ of $N$ (see Theorem 2.8 for the 
definition of the Kähler strata). Moreover, the manifold $Y_{0}$ is obtained as a hyperkähler quotient of a certain open subset of $T^{*} V$. The hyperkähler quotient metric on $Y_{0}$ coincides with the Feix-Kaledin metric.

Before going to the proof of Theorem 3.4 we need to state several auxiliary results. The next two lemmas are concerned with descent of group actions to quotients.

Lemma 3.5: Let $S \in O b(C)$ be a complex variety in the algebraic or analytic category equipped with an action of a reductive group $G$. Suppose also that a group $H$ acts on $S$ algebraically (resp. holomorphically) and the action of $H$ commutes with $G$. Assume that there exists the good quotient $T=S / G$. Then the action of $H$ on $S$ descends uniquely to an action of $H$ on $T$ in such a way that the quotient map is $H$-equivariant.

Proof: We shall prove the lemma in the algebraic case, the proof in the complex analytic case is similar. As the good quotient map is by definition affine it is enough to prove the statement in the case when $S$ is affine i.e. $S=$ $\operatorname{Spec}(A)$ where $A$ is a $\mathbb{C}$-algebra of finite type. In that case $T=\operatorname{Spec}\left(A^{G}\right)$ by Proposition 2.15. As the action of $H$ commutes with the action of $G$ the subalgebra $A^{G}$ is preserved by the $H$-action on $A$. This action on $A^{G}$ induces the action of $H$ on $T$.

Lemma 3.6: Let $M$ be a Kähler manifold and $X$ be the total space of the cotangent bundle to $M$. Suppose that $X$ admits the globally defined FeixKaledin hyperkähler metric. Suppose that $M$ is equipped with a Hamiltonian action of a compact Lie group $C$. Consider the natural lifting of this action to $X$. Fix a hyperkähler moment map $\mu_{h k}: X \rightarrow \mathfrak{c}^{*} \otimes \mathbb{R}^{3}$ on $X$ of the form

$$
\mu_{h k}=\left(\mu_{I}, \operatorname{Re} m, \operatorname{Im} m\right)
$$

with $m$ as in Proposition 2.23 and $\mu_{I}$ an arbitrary moment map for $\omega_{I}$. Consider the $U(1)$-action on $X$ by fiberwise multiplication. Then $\mu^{-1}(0)$ is $U(1)$ invariant. Moreover, the action of $U(1)$ on $\mu^{-1}(0)$ descends to the action of $U(1)$ on the hyperkähler quotient $X / / / C$ which is HKLR-compatible with the hyperkähler metric.

Proof: It is a classical fact. From the explicit description of $m$ (Proposition 2.23) we notice that $m^{-1}(0)$ is $U(1)$-invariant. Hence the $U(1)$-invariance of $\mu_{h k}^{-1}(0)$ will follow from the $U(1)$-invariance of $\mu_{I}$.

For every $\lambda \in U(1)$ the function $\lambda^{*} \mu_{I}$ is still a moment map for the action of $C$. Indeed, for every $\xi \in \mathfrak{c}$

$$
\left.\left.d\left\langle\lambda^{*} \mu, \xi\right\rangle=\lambda^{*}\langle d \mu, \xi\rangle=\lambda^{*}(\xi\lrcorner \omega_{I}\right)=\xi\right\lrcorner \omega_{I}
$$

as the vector field $\xi$ and the 2 -form $\omega_{I}$ are both $U(1)$-invariant. Hence the functions $\lambda^{*} \mu_{I}$ and $\mu_{I}$ differ by a constant. As they are certainly equal on $M$ they are also equal on $X$. 
The $U(1)$-action commutes with the $C$-action, thereby descending to the action of $U(1)$ on the quotient $X / / C$. Let $\widetilde{\omega_{I}}$ and $\widetilde{\Omega}$ denote the Kähler form and the holomorphic 2 -form on the quotient. They are uniquely defined by the properties that $\rho^{*} \widetilde{\omega_{I}}=\left.\omega_{I}\right|_{\mu^{-1}(0)}$ and $\rho^{*} \widetilde{\Omega}=\left.\Omega\right|_{\mu^{-1}(0)}$ where $\rho: \mu^{-1}(0) \rightarrow X / / C$ is the quotient map. Hence the compatibility properties (Definition 1.1)

$$
\begin{aligned}
& \lambda^{*} \omega_{I}=\omega_{I} \\
& \lambda^{*} \Omega=\lambda \Omega
\end{aligned}
$$

are preserved by taking the quotient.

The following trivial lemma describes the $U(1)$-action on total spaces of vector bundles in terms of the $U(1)$-action on functions. We shall omit its proof.

Lemma 3.7: Let $E=\operatorname{Spec}_{M}\left(\mathrm{~S}^{\bullet} \mathcal{F}\right)$ be the total space of a vector bundle over a complex algebraic variety $M$ where $\mathcal{F}$ is the locally free sheaf on $M$ whose dual is isomorphic to the sheaf of sections of the bundle $E \rightarrow M$. Then the $U(1)$-action on $E$ by fiberwise multiplication is induced by the $U(1)$-action on the sheaf of algebras $\mathrm{S} \bullet \mathcal{F}$ given by

$$
\lambda \cdot s=\lambda^{-r} s
$$

for $\lambda \in U(1) \subset \mathbb{C}^{\times}$and $s$ a local section of $\mathrm{S}^{r} \mathcal{F}$.

We are now ready to give a proof of Theorem 3.4.

Proof. Step 1: Consider the set

$$
V^{\circ}:=\left\{x \in V \mid x \in V^{s} \text { and } \operatorname{Stab}_{G}(x):=\{\mathrm{id}\}\right\}
$$

By Proposition 2.31 the geometric quotient of $V^{\circ}$ exists and is isomorphic to the unique open Kähler stratum $N^{\circ}$ in $N:=V / / C$. Let $X^{\circ}:=T^{*} V^{\circ}$ be the total space of cotangent bundle to $V^{\circ}$ and $Z^{\circ}:=\left\{x \in X^{\circ} \mid m(x)=0\right\}$. Proposition 2.24 applied to the free action of $G$ on $V^{\circ}$ tells us that $Y^{\circ}:=T^{*} N^{\circ}$ is isomorphic to $X^{\circ} / / G=Z^{\circ} / G$ as a complex manifold. By Corollary 3.3 all the points of $Z^{\circ}$ are stable. Hence by Proposition $2.31 Z^{\circ} / G$ is isomorphic to the Kähler quotient $Z^{\circ} / / C=X^{\circ} / / C$ as a complex manifold. One has the following 
commutative diagram.

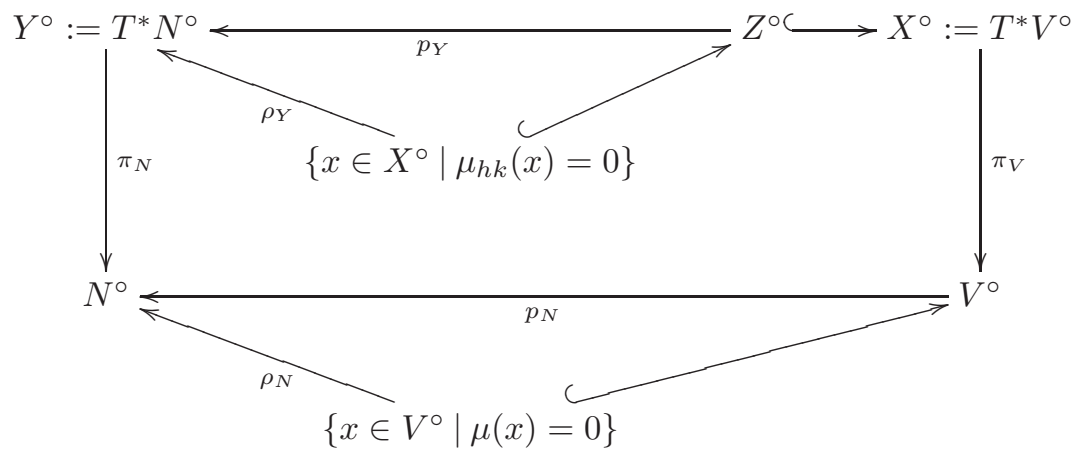

The manifold $Y^{\circ}$ is a hyperkähler quotient of $X^{\circ}$. Hence $Y^{\circ}$ admits a hyperkähler metric (Theorem 2.6). It remains to prove that the constructed metric is indeed the Feix-Kaledin metric.

Step 2: Let $\widetilde{\Omega} \in \Lambda_{I}^{2,0}\left(Y^{\circ}\right)$ be the holomorphic symplectic form on $Y^{\circ}$ induced by the hyperkähler structure. Equivalently, this is the 2-form on $Y^{\circ}$ whose pullback to $Z^{\circ}$ by the quotient map $p_{Y}$ is $\left.\Omega\right|_{Z^{\circ}}$. Here $\Omega$ stands as before for the standard holomorphic symplectic form on $X^{\circ}$. Such a 2 -form exists by Proposition 2.25. We need to prove that $\widetilde{\Omega}$ is the standard holomorphic symplectic form on the total space of the cotangent bundle.

Consider the restriction of the tautological 1-form $\tau$ on $X^{\circ}$ to $Z^{\circ}$. In Proposition 2.23 we proved that $\left.\tau\right|_{Z^{\circ}}$ is $G$-invariant and that $\left.\tau\right|_{Z^{\circ}}(\xi)=\left\langle\left. M\right|_{Z^{\circ}}, \xi\right\rangle=$ 0 for every $\xi \in \mathfrak{g}$. Hence by Proposition $\left.2.25 \tau\right|_{z^{\circ}}$ descends to a 1 -form on $Y^{\circ}=Z^{\circ} / G$. We shall denote this 1 -form by $\widetilde{\tau}$. Now take a point $x \in V^{\circ}$ and some $\alpha \in \operatorname{Ann}\left(\mathfrak{g}_{x}\right) \subset T_{x}^{*} V$. The covector $\alpha$ can be seen as a point in $Z^{\circ}$. Denote by $\widetilde{x}$ and $\widetilde{\alpha}$ the images of $x$ and $\alpha$ under the projection $p_{Y}: Z^{\circ} \rightarrow Y^{\circ}$. Consider any vector $v \in T_{\alpha} Z^{\circ}$. Then

$$
\widetilde{\tau}_{\widetilde{\alpha}}\left(p_{Y *} v\right)=\tau_{\alpha}(v)=\alpha\left(\pi_{V *} v\right)=\widetilde{\alpha}\left(p_{N *} \pi_{V *} v\right)=\widetilde{\alpha}\left(\pi_{N *} p_{Y *} v\right)
$$

where the first and the third identity hold as $\left.\tau\right|_{z^{\circ}}=p_{Y}^{*} \widetilde{\tau}$ and $\alpha=p_{N}^{*} \widetilde{\alpha}$ as a covector. The second identity follows from the definition of $\tau$. We obtain that $\widetilde{\tau}$ is the tautological 1-form on $Y^{\circ}=T^{*} N^{\circ}$. By the construction of the 2-form $\widetilde{\Omega}$ it is equal to $-d \widetilde{\tau}$. We see that $\widetilde{\Omega}$ is indeed the standard 2 -form on $Y^{\circ}$.

Step 3: Now we shall prove that the $U(1)$-action on $Y^{\circ}$ by fiberwise multiplication is HKLR-compatible with the constructed hyperkähler metric on $Y^{\circ}$.

By applying Lemma 3.6 to $M=V^{\circ}$ we introduce a $U(1)$-action on $Y^{\circ}=$ $X^{\circ} / / / C$ which is HKLR-compatible with the hyperkähler metric. When we apply Lemma 3.5 to the case of $S=Z^{\circ}, H=U(1)$ we also obtain a $U(1)$-action on $Y^{\circ}=Z^{\circ} / G$. These two actions coincide as the inclusion of $\mu_{h k}^{-1}(0)$ to $Z^{\circ}$ is $U(1)$-equivariant.

One can see that

$$
Y^{\circ} \cong \operatorname{Spec}_{N^{\circ}}\left(\mathrm{S}^{\bullet}\left(\mathcal{T} N^{\circ}\right)\right) \cong \operatorname{Spec}_{N^{\circ}}\left(S^{\bullet}\left(p_{*}\left(\mathcal{T} V^{\circ} / \mathfrak{g} \cdot\left(\mathcal{O}_{V^{\circ}}\right)\right)^{G}\right)\right.
$$


Here the first isomorphism holds as $Y^{\circ}$ is by definition the total space of the cotangent bundle to $N^{\circ}$. The second isomorphism follows from Proposition 2.25. With these isomorphisms in mind we use Lemma 3.5 and Lemma 3.7 to see that the $U(1)$-action on $Y^{\circ}=T^{*} N^{\circ}$ constructed above is the one given by the fiberwise multiplication. We finally proved Theorem 3.4

Remark 3.8: Theorem 3.4 can be stated without the assumption that the action of $C$ on $V$ is generically free as follows.

Theorem 3.9: Let $V$ be an Hermitian vector space. Suppose that a complex reductive group $G$ acts on $V$ linearly and the restriction of the action of $G$ to some fixed maximal compact subgroup $C \subset G$ preserves the Hermitian structure. Let $N:=V / / C$ be the Kähler quotient of $V$ by $C$ with respect to a fixed moment map $\mu: V \rightarrow \mathfrak{c}^{*}$. Then the Feix-Kaledin metric is globally defined on the total space $Y_{i}$ of the cotangent bundle to every Kähler stratum $N_{i}$ of $N$ (see Theorem 2.8 for the definition of the Kähler strata).

Theorem 3.9 follows easily from Theorem 3.4 as the closure of every Kähler stratum $N_{i} \subset N$ is itself a Kähler quotient of a vector space (Theorem 2.8).

\subsection{Metric completions of cotangent bundles}

Let $N$ be again a Kähler quotient of an Hermitian vector space $V$. Assume that $N$ is smooth i.e. $N=N^{\circ}$ in the notation of the proof of Theorem 3.4. The manifold $Y:=T^{*} N$ admits a hyperkähler metric by Theorem 3.4.

Theorem 3.10: The metric completion $\widetilde{Y}$ of $Y$ has a structure of a stratified hyperkähler space (in the sense of [31]).

Proof: Consider the hyperkähler quotient $X / / / C$. As $X^{\circ}:=T^{*} V^{\circ}$ is a dense open subset of $X:=T^{*} V$ we see that $Y=X^{\circ} / / C$ embeds into $X / / C$ as a dense open subset and the embedding preserves stratified hyperkähler structures. It is clear that $X$ is a complete metric space. Hence $X / / / C$ is itself a complete metric space as a quotient of the complete metric space $\mu_{h k}^{-1}(0) \subset X$ by a compact group $C$. Hence $X / / / C$ is isomorphic to the metric completion of $Y$.

Remark 3.11: In general the space $Y$ is not itself metrically complete. The cases when $Y$ is indeed complete were classified in [4] for $C$ being a torus. By the results of [4] the space $Y=T^{*} N$ is complete if and only if $N$ is isomorphic to a product of complex projective spaces. The question of completeness will be discussed further in Section 4.3.

\subsection{Example: Hirzebruch surfaces}

We shall now illustrate Theorem 3.4 and Theorem 3.10 with a specific example. 
Table 1: Unstable subspaces of $\mathbb{C}^{4}$

\begin{tabular}{|c|c|c|c|c|}
\hline Inequalities on $a$ and $b$ & \multicolumn{3}{|c|}{ Signs of the eigenvalues of $-\sqrt{-1} \xi$} & $\left(\mathbb{C}^{4}\right)^{\xi \geq 0}$ \\
& $a$ & $b$ & $b-n a$ & \\
\hline$a \geq 0>b$ & $\geq 0$ & $<0$ & $<0$ & $\left\{\left(x_{0}, x_{1}, 0,0\right\}\right.$ \\
\hline$b \geq 0>a$ & $<0$ & $\geq 0$ & $>0$ & $\left\{\left(0,0, y_{0}, y_{1}\right)\right\}$ \\
\hline $0>b \geq n a$ & $<0$ & $<0$ & $\geq 0$ & $\left\{\left(0,0,0, y_{1}\right)\right\}$ \\
\hline $0>n a>b$ & $<0$ & $<0$ & $<0$ & $\{(0,0,0,0)\}$ \\
\hline
\end{tabular}

Definition 3.12: Fix a positive integer $n$. Let the 2-dimensional torus $G=$ $\mathbb{C}^{\times} \times \mathbb{C}^{\times}$act on $\mathbb{C}^{4}$ via

$$
(\lambda, \mu) \cdot\left(x_{0}, x_{1}, y_{0}, y_{1}\right):=\left(\lambda x_{0}, \lambda x_{1}, \mu y_{0}, \mu \lambda^{-n} y_{1}\right)
$$

The quotient of $\mathbb{C}^{2} \backslash\{0\} \times \mathbb{C}^{2} \backslash\{0\} \subset \mathbb{C}^{4}$ by this action is called the Hirzebruch surface $\Sigma_{n}$.

Hirzebruch surfaces are ruled surfaces over $\mathbb{P}^{1}$. Indeed, consider the map $\Sigma_{n} \rightarrow \mathbb{P}^{1}$ which sends a point of $\Sigma_{n}$ represented by a point $\left(x_{0}, x_{1}, y_{0}, y_{1}\right) \in$ $\mathbb{C}^{2} \backslash\{0\} \times \mathbb{C}^{2} \backslash\{0\}$ to $\left[x_{0}: x_{1}\right] \in \mathbb{P}^{1}$. One can see that the fibers of this map are isomorphic to $\mathbb{P}^{1}$.

Our first goal is to represent the Hirzebruch surfaces as Kähler quotients. This is done as follows. Consider the action of $C=U(1) \times U(1)$ on $\mathbb{C}^{4}$ which is the restriction of the action of $\mathbb{C}^{\times} \times \mathbb{C}^{\times}$as in formula (3.5) to $C \subset G$. By formula (2.23) the moment map $\mu: \mathbb{C}^{4} \rightarrow \mathfrak{c}^{*}=\mathbb{R}^{2}$ is given by

$$
\mu(x, y)=-\frac{1}{2}\left(\|x\|^{2}-n\left|y_{1}\right|^{2}-c_{0},\|y\|^{2}-c_{1}\right)
$$

where $x=\left(x_{0}, x_{1}\right) \in \mathbb{C}^{2}, y=\left(y_{0}, y_{1}\right) \in \mathbb{C}^{2}$ and $c_{0}, c_{1}$ are some real numbers. From now on we assume that $c_{0}$ and $c_{1}$ are both positive.

Let us now describe the sets of stable and semistable points of $\mathbb{C}^{4}$. Recall that by Corollary 2.39 they are equal to

$$
V^{s}=V \backslash\left(\bigcup_{\langle\theta, \xi\rangle \leq 0} V^{\xi \geq 0}\right) ; \quad V^{s s}=V \backslash\left(\bigcup_{\langle\theta, \xi\rangle<0} V^{\xi \geq 0}\right)
$$

where the unions are taken over non-zero $\xi=(a, b) \in \mathfrak{c}=\mathbb{R}^{2}$. In our case $\theta \in \mathfrak{c}^{*}=\mathbb{R}^{2}$ is equal to $\frac{1}{2}\left(c_{0}, c_{1}\right)$. The inequality $\langle\theta, \xi\rangle=\frac{1}{2}\left(c_{0} a+c_{1} b\right) \leq 0$ implies that either $a$ or $b$ is negative. The element $\xi=(a, b)$ of the Lie algebra $\mathfrak{c}$ acts on $\mathbb{C}^{4}$ via the linear operator $\sqrt{-1} \operatorname{diag}(a, a, b, b-n a)$ by formula (3.5). Table 1 describes the subspaces $\left(\mathbb{C}^{4}\right)^{\xi \geq 0}$ for all possible $\xi=(a, b)$ such that $\langle\theta, \xi\rangle \leq 0$, according to inequalities on $a$ and $b$.

One can see from Table 1 and formula (3.7) that the sets of stable and semistable points coincide and are as follows:

$$
\left(\mathbb{C}^{4}\right)^{s}=\left(\mathbb{C}^{4}\right)^{s s}=\mathbb{C}^{4} \backslash\left(\left\{\left(x_{0}, x_{1}, 0,0\right)\right\} \cup\left\{\left(0,0, y_{0}, y_{1}\right)\right\}=\mathbb{C}^{2} \backslash\{0\} \times \mathbb{C}^{2} \backslash\{0\}\right.
$$


Table 2: Unstable subspaces of $T^{*} \mathbb{C}^{4}$

\begin{tabular}{|l|c|c|c|c|c|c|c|}
\hline $\begin{array}{l}\text { Inequalities } \\
\text { on } a \text { and } b\end{array}$ & \multicolumn{6}{|l|}{ Signs of the eigenvalues of $-\sqrt{-1} \xi$} & \\
& $a$ & $b$ & $\begin{array}{l}b- \\
n a\end{array}$ & $-a$ & $-b$ & $n a-$ & \\
& & & $n$ & & \\
\hline$a>0>b$ & $>0$ & $<0$ & $<0$ & $<0$ & $>0$ & $>0$ & $\left\{\left(x_{0}, x_{1}, 0,0,0,0, w_{0}, w_{1}\right)\right\}$ \\
\hline$a=0>b$ & $=0$ & $<0$ & $<0$ & $=0$ & $>0$ & $>0$ & $\left\{\left(x_{0}, x_{1}, 0,0, z_{0}, z_{1}, w_{0}, w_{1}\right)\right\}$ \\
\hline$b>0>a$ & $<0$ & $>0$ & $>0$ & $>0$ & $<0$ & $<0$ & $\left\{\left(0,0, y_{0}, y_{1}, z_{0}, z_{1}, 0,0\right)\right\}$ \\
\hline$b=0>a$ & $<0$ & $=0$ & $>0$ & $>0$ & $=0$ & $<0$ & $\left\{\left(0,0, y_{0}, y_{1}, z_{0}, z_{1}, w_{0}, 0\right)\right\}$ \\
\hline $0>n a>b$ & $<0$ & $<0$ & $<0$ & $>0$ & $>0$ & $>0$ & $\left\{\left(0,0,0,0, z_{0}, z_{1}, w_{0}, w_{1}\right)\right\}$ \\
\hline $0>n a=b$ & $<0$ & $<0$ & $=0$ & $>0$ & $>0$ & $=0$ & $\left\{\left(0,0,0, y_{1}, z_{0}, z_{1}, w_{0}, w_{1}\right)\right\}$ \\
\hline $0>b>n a$ & $<0$ & $<0$ & $>0$ & $>0$ & $>0$ & $<0$ & $\left\{\left(0,0,0, y_{1}, z_{0}, z_{1}, w_{0}, 0\right)\right\}$ \\
\hline
\end{tabular}

Proposition 2.31 implies that the Kähler quotient $\mathbb{C}^{4} / / C$ is the Hirzebruch surface $\Sigma_{n}$.

We notice that the stabilizer of every point of $\left(\mathbb{C}^{4}\right)^{s}=\mathbb{C}^{2} \backslash\{0\} \times \mathbb{C}^{2} \backslash\{0\}$ is trivial. Hence the set $\left(\mathbb{C}^{4}\right)^{\circ}$ of stable points with trivial stabilizer considered in the proof of Theorem 3.4 coincides with $\left(\mathbb{C}^{4}\right)^{s}$.

If one wants to construct the variety $T^{*} \Sigma_{n}$ one should take the hyperkähler quotient $T^{*}\left(\mathbb{C}^{4}\right)^{\circ} / / C$. Our second goal in this subsection is to show that the variety $T^{*} \Sigma_{n}=T^{*}\left(\mathbb{C}^{4}\right)^{\circ} / / G$ is strictly contained in the hyperkähler quotient $T^{*}\left(\mathbb{C}^{4}\right) / / / C$ and to describe the complement of $T^{*} \Sigma_{n}$ in $T^{*}\left(\mathbb{C}^{4}\right) / / / C$.

The action of $G=\mathbb{C}^{\times} \times \mathbb{C}^{\times}$on $T^{*} \mathbb{C}^{4}$ is given by

$$
\begin{aligned}
& (\lambda, \mu) \cdot\left(x_{0}, x_{1}, y_{0}, y_{1}, z_{0}, z_{1}, w_{0}, w_{1}\right)= \\
& \quad=\left(\lambda x_{0}, \lambda x_{1}, \mu y_{0}, \mu \lambda^{-n} y_{1}, \lambda^{-1} z_{0}, \lambda^{-1} z_{1}, \mu^{-1} w_{0}, \mu^{-1} \lambda^{n} w_{1}\right)
\end{aligned}
$$

We want to describe the sets of stable and semistable points of $T^{*} \mathbb{C}^{4}$. This problem is solved analoguously to the problem of description of stable and semistable points of $\mathbb{C}^{4}$. The element $\xi=(a, b)$ of the Lie algebra $\mathfrak{c}$ acts on $T^{*} \mathbb{C}^{4}$ via the linear operator $\sqrt{-1} \operatorname{diag}(a, a, b, b-n a,-a,-a,-b, n a-b)$ by formula (3.9). Table 2 describes the subspaces $\left(T^{*} \mathbb{C}^{4}\right)^{\xi \geq 0}$ for all possible $\xi=(a, b)$ such that $\langle\theta, \xi\rangle \leq 0$, according to inequalities on $a$ and $b$.

The set of unstable points is the union of vector subspaces $\left(T^{*} \mathbb{C}^{4}\right)^{\xi \geq 0}$ in the last column of Table 2. One can see from formula (3.7) that the sets of stable and semistable points of $T^{*} \mathbb{C}^{4}$ coincide and

$$
\begin{aligned}
T^{*} \mathbb{C}^{4} \backslash\left(T^{*} \mathbb{C}^{4}\right)^{s}=\left(T^{*} \mathbb{C}^{4}\right)^{u s}= & \\
=\left\{\left(x_{0}, x_{1}, 0,0, z_{0}, z_{1}, w_{0}, w_{1}\right)\right\} \cup & \left\{\left(0,0, y_{0}, y_{1}, z_{0}, z_{1}, w_{0}, 0\right)\right\} \cup \\
& \cup\left\{\left(0,0,0, y_{1}, z_{0}, z_{1}, w_{0}, w_{1}\right)\right\}
\end{aligned}
$$

Consider the set $E$ of points of $T^{*} \mathbb{C}^{4}$ which are (semi)stable but whose images in $\mathbb{C}^{4}$ under the natural projection $p$ to $\mathbb{C}^{4}$ are not (semi)stable. Of course, it does 
not matter if one considers stability or semistability because in our case these notions coincide. By formula (3.8) and formula (3.10) the set $E$ is non-empty and is described as follows:

$$
\begin{aligned}
E=\left(p^{-1}\left(\mathbb{C}^{4}\right)^{u s}\right) \backslash & \left(T^{*} \mathbb{C}^{4}\right)^{u s}= \\
& =\left\{\left(0,0, y_{0}, y_{1}, z_{0}, z_{1}, w_{0}, w_{1}\right) \mid y_{0} \neq 0 \text { and } w_{1} \neq 0\right\}
\end{aligned}
$$

Consider the set $Z^{s}$ of stable points of $T^{*} \mathbb{C}^{4}$ on which the holomorphic symplectic map $m: T^{*} \mathbb{C}^{4} \rightarrow \mathfrak{g}^{*}=\mathbb{C}^{2}$ vanishes. The set $Z^{s}$ contains a dense open subset $Z^{\circ}=\left\{x \in Z^{s} \mid p(x) \in\left(\mathbb{C}^{4}\right)^{s}=\left(\mathbb{C}^{4}\right)^{\circ}\right\}$. The quotient of $Z^{\circ}$ by $G$ is nothing but $T^{*} \Sigma_{n}=T^{*}\left(\mathbb{C}^{4}\right)^{\circ} / / C$. The complement of $T^{*} \Sigma_{n}$ in the hyperkähler quotient $T^{*} \mathbb{C}^{4} / / / C$ is thus isomorphic to the quotient of $Z^{s} \cap E$ by $G$ as a complex variety.

Let us first describe the variety $Z^{s} \cap E$. By Proposition 2.23 the moment $\operatorname{map} m: T^{*} \mathbb{C}^{4} \rightarrow \mathbb{C}^{2}$ is as follows:

$$
m\left(x_{0}, x_{1}, y_{0}, y_{1}, z_{0}, z_{1}, w_{0}, w_{1}\right)=\sqrt{-1}\left(x_{0} z_{0}+x_{1} z_{1}-n y_{1} w_{1}, y_{0} w_{0}+y_{1} w_{1}\right)
$$

Consider the restriction of $m$ to $E$. It is given as

$$
m\left(0,0, y_{0}, y_{1}, z_{0}, z_{1}, w_{0}, w_{1}\right)=\sqrt{-1}\left(-n y_{1} w_{1}, y_{0} w_{0}+y_{1} w_{1}\right)
$$

A point of $E$ thus lies in $Z^{s}$ if and only if $y_{0} w_{0}=y_{1} w_{1}=0$. By formula (3.11)

$$
E \cap Z^{s}=\left\{\left(0,0, y_{0}, 0, z_{0}, z_{1}, 0, w_{1}\right) \mid y_{0} \neq 0 \text { and } w_{1} \neq 0\right\}
$$

To describe the quotient of $E \cap Z^{s}$ by the action of $G$ we notice that every $G$-orbit of $E \cap Z^{s}$ intersects with the set $S:=\left\{y_{0}=w_{1}=1\right\}$. Two points $p=\left(0,0,1,0, z_{0}, z_{1}, 0,1\right)$ and $p^{\prime}=\left(0,0,1,0, z_{0}^{\prime}, z_{1}^{\prime}, 0,1\right)$ of $S$ lie in the same $G$ orbit if and only if $\left(z_{0}^{\prime}, z_{1}^{\prime}\right)=\lambda\left(z_{0}, z_{1}\right)$ for some $n$-th root of unity $\lambda$. Therefore

$$
\left(E \cap Z^{s}\right) / G=S / \mu_{n} \cong \mathbb{C}^{2} / \mu_{n}
$$

We have proved the following.

Proposition 3.13: The manifold $T^{*} \Sigma_{n}$ admits the globally defined FeixKaledin metric. This metric is non-complete. The metric completion of $T^{*} \Sigma_{n}$ is the hyperkähler quotient $\left(T^{*} \mathbb{C}^{4}\right) / / / C$. The complement of $T^{*} \Sigma_{n}$ in $\left(T^{*} \mathbb{C}^{4}\right) / / C$ is isomorphic to $\mathbb{C}^{2} / \mu_{n}$ as a complex variety. In particular, the underlying complex variety of $\left(T^{*} \mathbb{C}^{4}\right) / / / C$ is non-smooth and has quotient singularities.

\section{Cotangent Bundles as Stein and Affine Vari- eties}

\subsection{Twisted complex structure on cotangent bundle}

Consider the vector space $X_{\mathbb{R}}=V_{\mathbb{R}} \oplus V_{\mathbb{R}}$ as a real vector space with the complex structure $J$. Recall that

$$
J(x, y)=(-y, x)
$$


We draw the reader's attention to the fact that the (semi)stability of a point $x \in X$ in general does depend on the choice of a complex structure on $X$. In this subsection we will consider points of $X$ which are semistable with respect to the complex structure $J$, the Kähler form $\omega_{J}$ and the moment map $\mu_{J}=\operatorname{Re} m$. We shall call these points $J$-semistable.

Proposition 4.1: Every point of $X$ is $J$-semistable with respect to the moment $\operatorname{map} \mu_{J}=\operatorname{Re} m$.

Proof: We shall prove the statement using the Hilbert-Mumford criterion i.e. by computing the $\mu$-weights of points of $X$ with respect to the complex structure $J$ (Definition 2.33). For a point $(x, y) \in X=V_{\mathbb{R}} \oplus V_{\mathbb{R}}$ and $\xi \in \mathfrak{c}$ we shall denote the corresponding $\mu$-weight by $w_{J}^{\xi}(x, y)$. Let $\xi \in \mathfrak{c}$ be a skew-symmetric operator acting on $V$. Then the matrix of its action on $X$ is

$$
\xi_{X}=\left(\begin{array}{ll}
\xi & 0 \\
0 & \xi
\end{array}\right)
$$

and

$$
J \xi_{X}=\left(\begin{array}{cc}
0 & -\xi \\
\xi & 0
\end{array}\right)
$$

The exponent of $J \xi_{X}$ is given by

$$
\exp \left(t J \xi_{X}\right)=\left(\begin{array}{cc}
\cos t \xi & -\sin t \xi \\
\sin t \xi & \cos t \xi
\end{array}\right)
$$

Now let $\sqrt{-1} \lambda_{i}$ be (imaginary) eigenvalues of the operator $\xi$ as in Example 2.37. We can decompose every vector $x \in V$ into the sum $x=\sum x_{i}$ of eigenvectors of $\xi$. If we consider $V$ as a real vector space with the complex structure $I$ then

$$
\xi x_{i}=\lambda_{i} I x_{i}
$$

hence

$$
\cos (t \xi) x_{i}=\cosh \left(t \lambda_{i}\right) x_{i}, \quad \sin (t \xi) x_{i}=\sinh \left(t \lambda_{i}\right) I x_{i}
$$

Let

$$
(x(t), y(t)):=\exp \left(t J \xi_{X}\right)(x, y)
$$

Then due to formulae (4.2) and (4.3)

$$
\begin{aligned}
& x(t)=\sum_{i} \cosh \left(t \lambda_{i}\right) x_{i}-I \sum_{i} \sinh \left(t \lambda_{i}\right) y_{i} \\
& y(t)=I \sum_{i} \sinh \left(t \lambda_{i}\right) x_{i}+\sum_{i} \cosh \left(t \lambda_{i}\right) y_{i}
\end{aligned}
$$

By Definition 2.33 and formula (2.12) the $\mu$-weights are equal to

$$
w_{J}^{\xi}(x, y)=\lim _{t \rightarrow \infty}\langle\xi x(t), y(t)\rangle
$$


We now use formula (4.7) to compute them explicitly. The vector $\xi x(t)$ is equal to

$$
\xi x(t)=I \sum_{i} \lambda_{i} \cosh \left(t \lambda_{i}\right) x_{i}+\sum_{i} \lambda_{i} \sinh \left(t \lambda_{i}\right) y_{i}
$$

Hence the scalar product of $\xi x(t)$ with the vector $y(t)$ equals

$$
\sum_{i} \lambda_{i}\left[\frac{1}{2} \sinh \left(2 t \lambda_{i}\right)\left(\left\|x_{i}\right\|^{2}+\left\|y_{i}\right\|^{2}\right)+\cosh \left(2 t \lambda_{i}\right) \omega\left(x_{i}, y_{i}\right)\right]
$$

Computing the limit of the sum is an exercise in a real calculus. As long as $\lambda_{i} \neq 0$ and at least one of the vectors $x_{i}$ and $y_{i}$ is non-zero, the $i$-th summand tends either to $+\infty$ or to 0 . The last case occurs if and only if $x_{i}=\operatorname{sgn}\left(\lambda_{i}\right) I y_{i}$. In particular, $w_{J}^{\xi}(x, y)$ is always non-negative. By Hilbert-Mumford criterion (Theorem 2.35) every point of $X$ is $J$-semistable.

As a corollary of the proposition above we obtain the following

Theorem 4.2: Let $\widetilde{Y}=X / / / C$ be a hyperkähler quotient of $X=T^{*} V$. Let $J \in \mathbb{H}$ be a complex structure on $\widetilde{Y}$ different from $I$ and $-I$. Then the complex analytic variety $\widetilde{Y}_{J}$ is an affine algebraic variety. Moreover, for every two complex structures $J_{1}$ and $J_{2}$ different from $\pm I$ the varieties $\widetilde{Y}_{J_{1}}$ and $\widetilde{Y}_{J_{2}}$ are isomorphic as algebraic varieties.

Proof. Step 1: Let $J$ be the complex structure on the vector space $X$ as defined in formula (4.1). The action of $C$ on $X$ preserves $J$ and can be uniquely extended to the $J$-holomorphic action of the complexified group $G$ on $X_{J}$ as in formula (4.2). Consider the holomorphic symplectic form $\Omega_{J}=\omega_{K}+\sqrt{-1} \omega_{I} \in$ $\Lambda_{J}^{2,0} X$. It is preserved by the action of $C$ and hence by the $J$-holomorphic action of $G$. Indeed, for every $\xi \in \mathfrak{c}$

$$
\left.\left.\mathrm{L}_{J \xi} \Omega_{J}=d(J \xi\lrcorner \Omega_{J}\right)=\sqrt{-1} d(\xi\lrcorner \Omega_{J}\right)=\sqrt{-1} \mathrm{~L}_{\xi} \Omega_{J}=0
$$

as $\Omega_{J}$ is closed.

Consider the $J$-holomorphic moment map $m_{J}: X \rightarrow \mathfrak{g}^{*}$. Explicitly, for every $\xi \in \mathfrak{c}$

$$
\left\langle m_{J}, \xi\right\rangle=\langle\operatorname{Im} m, \xi\rangle+\sqrt{-1}\left\langle\mu_{I}, \xi\right\rangle
$$

where $\mu_{I}: X \rightarrow \mathfrak{c}^{*}$ is the moment for the action of $C$ with respect to the complex structure $I$.

By Proposition 4.1 every point of the closed algebraic $G$-invariant subvariety $m_{J}^{-1}(0)$ is $J$-semistable. Hence by Proposition Proposition 2.31

$$
\tilde{Y}_{J}=m_{J}^{-1}(0) / / \mu_{J} C=m_{J}^{-1}(0) / G
$$

As $m_{J}^{-1}(0)$ is affine its good quotient $\widetilde{Y}_{J}$ is again affine by Proposition 2.15.

Step 2: Consider the action of $\mathbb{C}^{\times}$on $X$ by the fiberwise multiplication. For every two quaternionic complex structures $J_{1}, J_{2}$ not equal to $\pm I$ there exists 
$\lambda \in \mathbb{C}^{\times}$such that $\lambda_{*} J_{1}=J_{2}([21])$. Hence $\lambda$ is a complex linear $C$-equivariant isomorphism between $\left(X, J_{1}\right)$ and $\left(X, J_{2}\right)$. Moreover, $\lambda$ is an isomorphism of holomorphic symplectic vector spaces. If $m_{i}$ denotes the holomorphic symplectic moment map on $X_{J_{i}}$ then one can check that $\lambda^{*} m_{2}=m_{1}([21])$. As a consequence $\lambda$ induces an algebraic $G$-eqivariant isomorphism of $\widetilde{Y}_{J_{i}}=m_{i}^{-1}(0) / G$, $i=1,2$.

\subsection{Stein structure}

Throughout this subsection we assume that the hyperkähler quotient $\widetilde{Y}=X / / C$ is smooth. We present here another approach to Theorem 4.2. This approach does not let us to prove that $\widetilde{Y}_{J}$ is affine but only that it is Stein. However, it is interesting in its own way and possibly can be applied in a more general situation.

Definition 4.3: ([10], Ch. I §6, Ch. VII §9) Let $M$ be a complex manifold. It is called Stein if one of the following equivalent conditions holds:

(i) $M$ admits a closed holomorphic embedding to an affine complex space.

(ii) There exists a smooth proper strictly plurisubharmonic function $\rho: M \rightarrow$ $\mathbb{R}_{\geq 0}$ (see the definition below).

Definition 4.4: An $\mathbb{R}$-valued $C^{2}$-function on a complex manifold $M$ is called strictly plurisubharmonic if $d d^{c} \rho$ is a strictly positive $(1,1)$-form.

Remark 4.5: A (possibly singular) Stein space can be defined as a complex space admitting a closed embedding into $\mathbb{C}^{N}$. An equivalent definition in terms of strictly plurisubharmonic functions also does exist ([13]). However, defining strictly plurisubharmonic functions in terms of the $\bar{\partial}$-operator seems to be a subtle question. Its discussion will lead us too far beyond our topic. That's why we prefer to impose a rather restrictive smoothness assumption in our exposition.

Remark 4.6: Affine varieties are Stein. The converse is of course not true. Even more, there exist examples of Stein algebraic varieties which are not affine ([18], Ch. VI, Ex. 3.2).

Following [21] we are going to construct a proper strictly plurisubharmonic function on $\widetilde{Y}_{J}$.

Lemma 4.7: The $U(1)$-action on $\widetilde{Y}_{I}$ is Hamiltonian.

Proof: Being linear, the $U(1)$-action on $X$ is Hamiltonian. In addition, it commutes with $C$. Hence by the technique of reduction in stages ([8], Part IX $\S 24.3)$ the $U(1)$-action stays Hamiltonian after taking the quotient. 
Proposition 4.8: Suppose that the Kähler quotient $N=V / / C$ is smooth and compact. Let $\psi$ be a moment map for the $U(1)$ action on $\widetilde{Y}$. Then $-\psi$ is a proper strictly plurisubharmonic function on $\widetilde{Y}_{J}$.

Proof. Step 1: Let $X$ be an arbitrary hyperkähler manifold equipped with a Hamiltonian $U(1)$-action rotating the complex structures. Denote by $\varphi$ the vector field tangent to the $U(1)$ action. Then

$$
\begin{gathered}
\mathrm{L}_{\varphi} \omega_{I}=0 \\
\mathrm{~L}_{\varphi} \omega_{J}=\omega_{K} \\
\mathrm{~L}_{\varphi} \omega_{K}=-\omega_{J}
\end{gathered}
$$

The moment map $\psi: X \rightarrow \mathbb{R}$ satisfies

$$
d \psi=\varphi\lrcorner \omega_{I}
$$

As in [21] we compute

$$
\left.\left.\left.d d_{J}^{c} \psi=d J d \psi=d J(\varphi\lrcorner \omega_{I}\right)=d((J \varphi)\lrcorner \omega_{I}\right)=d(\varphi\lrcorner \omega_{K}\right)=\mathrm{L}_{\varphi} \omega_{K}=-\omega_{J}
$$

Hence $-\psi$ is a Kähler potential for $X_{J}$, in particular, this function is strictly plurisubharmonic.

Step 2: The moment map $\Psi: X \rightarrow \mathbb{R}$ for the $U(1)$-action on $X=V_{\mathbb{R}} \oplus V_{\mathbb{R}}$ is given by

$$
\Psi(x, y)=-\frac{1}{2}\|y\|^{2}
$$

It is a non-positive function. By Lemma 4.7 it descends to a moment map $\psi$ on $\tilde{Y}$. Hence the function $\rho:=-\psi$ is non-negative and strictly plurisubharmonic. Now we need to check that $\rho$ is proper.

Fix a number $r \in \mathbb{R}, r>0$ and consider the closed set $\rho^{-1}([0, r]) \subset \widetilde{Y}$. It is the quotient by a compact group of the following closed subset of $X$

$$
K_{r}:=\left\{(x, y) \in X \mid \mu_{h k}(x, y)=0,-\Psi(x, y) \leq r\right\}
$$

In particular, for $(x, y) \in K_{r} \subset V_{\mathbb{R}} \oplus V_{\mathbb{R}}$

$$
\mu_{I}(x, y)=0
$$

and $\|y\|^{2} \leq 2 r$. By Lemma 3.1 for every $(x, y) \in K_{r}$ and $\xi \in \mathfrak{c}$

$$
|\langle\mu(x), \xi\rangle|=\left|\frac{\omega(x, \xi x)}{2}+\langle\theta, \xi\rangle\right|=\left|\frac{\omega(y, \xi y)}{2}\right| \leq \frac{1}{2}\|\xi\|_{V}\|y\|^{2} \leq r\|\xi\|_{V}
$$

where $\|\xi\|_{V}$ is the norm of $\xi$ as an operator on $V$. Hence the claim will follow if we prove that the moment map $\mu: V \rightarrow \mathfrak{c}^{*}$ is proper.

Step 3: The properness of $\mu$ follows straightforwardly from [37], Lemma 4.10. For the sake of completeness we include the proof here. 
Suppose $\left\{u_{n}\right\}$ is an unbounded sequence of vectors of $V$. We want to check that the sequence $\left\{\mu\left(u_{n}\right)\right\}$ is also unbounded. By passing to a subsequence we may assume that $\lim _{n \rightarrow \infty}\left\|u_{n}\right\|$ exists and is equal to $+\infty$. The subset of points of the form $g v$ where $g \in G$ and $\mu(v)=0$ is dense in $V$, hence without loss of generality we may and shall assume that the points $u_{n}=g_{n} v_{n}$ are of this form.

Fix a maximal torus $T \subset C$ with the Lie algebra $t$. The reductive group $G$ admits the polar decomposition $G=C \exp (\sqrt{-1} \mathrm{t}) C$. One may write $g_{n}=$ $k_{n} \exp \left(\sqrt{-1} \xi_{n}\right) h_{n}$ where $k_{n}, h_{n} \in C$ and $\xi_{n} \in \mathfrak{t}$. As the subset $\mu^{-1}(0)$ is $C$ invariant one may assume that $h_{n}=1$ by changing $v_{n}$ if necessary. As the action of $C$ on $V$ changes neither the norm of a vector nor the norm of its image under $\mu$ one may assume that $k_{n}$ is also the unit element. Hence we are reduced to the case when $u_{n}=\exp \left(\sqrt{-1} \xi_{n}\right) v_{n}$ where $\xi_{n} \in \mathfrak{t}$ and $\mu\left(v_{n}\right)=0$.

Take any orthonormal basis $\left\{e^{i}\right\}$ of $V$ in which the action of $T$ is diagonalized. Then there exist $\beta^{i} \in \mathfrak{t}^{*}$ such that for every $\xi \in \mathfrak{t}$

$$
\xi \cdot e^{i}=\sqrt{-1} \beta^{i}(\xi) e^{i}
$$

Decompose $v_{n}=\sum_{i} v_{n}^{i} e^{i}$ with respect to this basis. Then

$$
\left\|g_{n} v_{n}\right\|^{2}=\sum_{i} \exp \left(-2 \beta^{i}\left(\xi_{n}\right)\right)\left|v_{n}^{i}\right|^{2}
$$

As by our assumptions the sequence $\left\|g_{n} v_{n}\right\|^{2}$ is unbounded, the subset of indices $A:=\left\{i \mid\right.$ the sequence $\exp \left(-2 \beta^{i}\left(\xi_{n}\right)\right)\left|v_{n}^{i}\right|^{2}$ is unbounded $\}$ is non-empty. By passing to a subsequence if necessary one may assume that $\forall i \in A$ the limit $\lim _{n \rightarrow \infty} \exp \left(-2 \beta^{i}\left(\xi_{n}\right)\right)\left|v_{n}^{i}\right|^{2}$ exists and is equal to $+\infty$. The set $A$ might become smaller but it will remain non-empty.

We assumed that the quotient $V / / C$ is compact. That is equivalent to the compactness of $\mu^{-1}(0)$. Hence $\left|v_{n}^{i}\right|^{2}$ is bounded $\forall i$. Consequently, $\beta^{i}\left(\xi_{n}\right)$ tends to $-\infty$ for every $i \in A$. In particular $\exists \eta \in \mathfrak{t}$ of unit norm such that $\forall i \in A$

$$
\beta^{i}(\eta)<0
$$

Now one can estimate

$$
\| \mu\left(g_{n} v_{n}\right)|| \geq\left|\left\langle\mu\left(g_{n} v_{n}\right), \eta\right\rangle\right|=\left.\left|\frac{1}{2} \sum_{i} \beta^{i}(\eta) \exp \left(-\beta^{i}\left(\xi_{n}\right)\right)\right| v_{n}^{i}\right|^{2}+\langle\theta, \eta\rangle \mid
$$

for some $\theta \in \mathfrak{c}^{*}$ depending only on the choice of $\mu$. By the choice of $\eta$ every summand in $\sum_{i} \beta^{i}(\eta) \exp \left(-\beta^{i}\left(\xi_{n}\right)\right)\left|v_{n}^{i}\right|^{2}$ is either bounded or tends to minus infinity. Hence $\mu\left(g_{n} v_{n}\right)$ is unbounded.

\subsection{Big and nef tangent bundles}

Let $N$ be a Kähler manifold and $E$ a vector bundle over $N$. The space of all hyperplanes in $E$ will be called the projectivization of $E$ and denoted by 
$\mathbb{P}(E)$. Clearly the manifold $\mathbb{P}(E)$ is isomorphic to $\operatorname{Tot}\left(E^{*}\right) / \mathbb{C}^{\times}$. There is the line bundle $\mathcal{O}(1)$ over $\mathbb{P}(E)$ defined similarly to the line bundle $\mathcal{O}(1)$ over a projective space.

\section{Definition 4.9:}

(i) A line bundle $L$ over $N$ is called a nef line bundle if its first Chern class $c_{1}(L) \in H^{1,1}(X, \mathbb{Z})$ lies in the closure of the Kähler cone of $N$.

(ii) A line bundle $L$ over $N$ is called a big line bundle if

$$
h^{0}\left(N, L^{\otimes d}\right)=O\left(d^{n}\right)
$$

where $n=\operatorname{dim} N$.

(iii) A vector bundle $E$ over $N$ is called a nef (resp. big) vector bundle if the line bundle $\mathcal{O}(1)$ over $\mathbb{P}(E)$ is a nef (resp. big) line bundle.

To determine whether a given vector bundle $E$ is nef (or big) we need some information about the manifold $\mathbb{P}(E)$. The next lemma is concerned with the case $E=T N$. Let us fix notation before stating the lemma. For any manifold $Z$ of dimension $m$ (resp. a smooth map $f: Z \rightarrow Z^{\prime}$ with fibers of equal dimension $m$ ) let $K_{Z}:=\Lambda^{m} \Omega_{Z}$ (resp. $\left.K_{Z / Z^{\prime}}:=\Lambda^{m} \Omega_{Z / Z^{\prime}}\right)$ denote the canonical line bundle of $Z$ (resp. the relative canonical line bundle of the map $f: Z \rightarrow Z^{\prime}$ ).

Lemma 4.10: Let $N$ be a complex manifold of dimension $n$. Then the canonical line bundle $K_{\mathbb{P}(T N)}$ on $\mathbb{P}(T N)$ is isomorphic to the line bundle $\mathcal{G}(-n)$.

Proof: Let $Z$ denote the manifold $\mathbb{P}(T N)$ and $\pi: Z \rightarrow N$ the natural projection. First, one has the relative Euler exact sequence of sheaves on $Z$

$$
0 \rightarrow \Omega_{Z / N} \rightarrow \pi^{*} \mathcal{T} N \otimes \mathcal{O}(-1) \rightarrow \mathcal{O}_{Z} \rightarrow 0
$$

By taking the highest exterior degree of the terms of the sequence we get

$$
\pi^{*} K_{N}^{*} \otimes \mathcal{O}(-n) \cong K_{Z / N}
$$

Second, we have the short exact sequence of differentials

$$
0 \rightarrow \pi^{*} \Omega_{N} \rightarrow \Omega_{Z} \rightarrow \Omega_{Z / N} \rightarrow 0
$$

hence

$$
K_{Z}=\pi^{*} K_{N} \otimes K_{Z / N}
$$

By putting formulae (4.18) and (4.20) together we obtain that

$$
K_{Z} \cong \pi^{*} K_{N} \otimes \pi^{*} K_{N}^{*} \otimes \mathcal{O}(-n) \cong \mathcal{O}(-n)
$$


Before going any further we shall state a part of [36], Prop. 1.10 in a form convenient for our exposition. This result will turn out to be useful to study the geometry of the manifold $Z=\mathbb{P}(T N)$.

Proposition 4.11: ([36]) Let $Y$ be a Kähler Ricci-flat manifold equipped with a Hamiltonian action of $U(1)$. Fix a moment map $\psi_{t_{0}}: Y \rightarrow \mathbb{R}$ and for any $t \in \mathbb{R}$ let $\psi_{t}:=\psi_{t_{0}}+\left(t-t_{0}\right)$ be another moment map. Assume that 0 is a regular value of $\psi_{t_{0}}$. For any point $y \in \psi^{-1}(0)$ consider a neighbourhood $Y^{\prime} \subset Y$ of $y$. Let us call points of $Y^{\prime}$ equivalent if they lie in the same $\mathbb{C}^{\times}$-orbit. Assume that $Y^{\prime}$ satisfies the following property: for any $t \in \mathbb{R}$ close enough to zero the set $\psi_{t}^{-1}(0)$ is contained in $Y^{\prime}$ and the natural map

$$
\psi_{t}^{-1}(0) / U(1) \rightarrow Y^{\prime} / \sim
$$

is an isomorphism. Let $\omega(t)$ be the Kähler form on $Z:=Y^{\prime} / \sim$ obtained from Kähler reduction with respect to $\psi_{t}$. Then the family $\omega(t)$ of forms satisfies the following differential equation

$$
c \frac{d}{d t} \omega(t)=\rho(t)-\sqrt{-1} \partial \bar{\partial} \log \delta(t)
$$

where $\rho(t)$ is the Ricci form of $\omega(t), \delta(z, t)$ is the length of the circle on the submanifold $\{\mu(x)+t=0\} \subset Y$ lying over a point $z \in Z$, and $c$ is a constant.

We shall now state a general theorem which relates the properties of the Feix-Kaledin metric on $Y=T^{*} N$ with the geometry of $N$. This theorem is due to R. Bielawski who kindly permitted me to write his proof here.

Theorem 4.12: (R. Bielawski, private correspondence) Let $N$ be a Kähler manifold of dimension $n$. Suppose that the Feix-Kaledin metric is defined globally on $Y=T^{*} N$. Assume that the moment map $\psi: Y \rightarrow \mathbb{R}$ for the $U(1)$-action on $Y$ is proper. Then the tangent bundle $T N$ to the manifold $N$ is nef.

Proof: Step 1: Choose the moment map $\psi: Y \rightarrow \mathbb{R}_{\leq 0}$ for the $U(1)$-action on $Y$ in such a way that $N=\psi^{-1}(0)$. For any $t>0$ let $\psi_{t}:=\psi+t$ be another moment map for the $U(1)$-action on $Y$. Denote by $Y / /{ }^{t} U(1)$ the Kähler quotient of $Y$ with respect to the moment map $\psi_{t}$. We start by showing that $Y /{ }^{t} U(1)$ is naturally isomorphic to $\mathbb{P}(T N)$ as a complex manifold. We do this by describing the set $Y_{t}^{s}$ of stable points of $Y$ with respect to the moment map $\psi_{t}$ with the use of the Hilbert-Mumford criterion (Theorem 2.35). We claim that for any $t>0$

$$
Y_{t}^{s}=Y \backslash N
$$

where $N$ is embedded to $Y$ as the zero section. Let $\xi \in \mathfrak{u}(1) \cong \mathbb{R}$ be the unit positive pointing vector. For any $x \in Y$ we shall denote by $w_{t}^{+}(x)\left(\operatorname{resp} . w_{t}^{-}(x)\right)$ the weight of the pair $x$ and $\xi$ (resp. $-\xi$ ) with respect to the moment map $\psi_{t}$ (Definition 2.33). We compute the weights:

$$
w_{t}^{+}(x)=\lim _{s \rightarrow \infty} \psi_{t}\left(e^{-s} x\right)=\lim _{s \rightarrow \infty} \psi\left(e^{-s} x\right)+t=t>0
$$


as $\lim _{s \rightarrow \infty} e^{-s} x$ is a point of the zero section and $\psi$ vanishes on the zero section.

$$
w_{t}^{-}(x)=-\lim _{s \rightarrow \infty} \psi_{t}\left(e^{s} x\right)=-\lim _{s \rightarrow \infty} \psi\left(e^{s} x\right)-t= \begin{cases}+\infty, & \text { if } x \notin N \\ -t<0, & \text { otherwise }\end{cases}
$$

Indeed, $\psi \leq 0$ and it cannot be bounded on the ray $\left\{e^{s} x\right\}$ as this map is proper. We obtain that $Y_{t}^{s}=Y \backslash N$ (Theorem 2.35). By Proposition 2.31 the good quotient $(Y \backslash N) / \mathbb{C}^{\times}$is isomorphic to the Kähler quotient $Y / /{ }^{t} U(1)$ as a complex variety. The quotient $(Y \backslash N) / \mathbb{C}^{\times}$is equal to $\mathbb{P}(T N)$ by the very definition of the projectivization of a vector bundle, hence the claim.

Step 2: In Step 1 we identified each Kähler quotient $Y / /{ }^{t} U(1)$ with $Z$. As $Y / /{ }^{t} U(1)$ is equipped with a Kähler form $\omega(t)$, we obtain a one-parameter family $\omega(t), t>0$ of Kähler forms on $Z$. The manifold $Y$ is Ricci-flat as it is hyperkähler. We may now apply Proposition 4.11 with $Y^{\prime}=Y^{s}=Y \backslash N$ to obtain

$$
c \frac{d}{d t} \omega(t)=\rho(t)-\sqrt{-1} \partial \bar{\partial} \log \delta(t)
$$

In [2], Prop. 1.2 Bielawski shows that if $Y$ is a hyperkähler manifold with a HKLR-compatible $U(1)$-action then $c=\operatorname{dim}_{H} Y=n$. As the Ricci form $\rho(t)$ represents the class of $c_{1}(Z)=-c_{1}\left(K_{Z}\right)$ one obtains that

$$
n \frac{d}{d t}[\omega(t)]=c_{1}(Z)
$$

By the previous step $c_{1}(Z)=n c_{1}(\Theta(1))$. Hence $[\omega(t)]=\left[\omega\left(t_{0}\right)\right]+(t-$ $\left.t_{0}\right) c_{1}(\Theta(1))$. Therefore

$$
c_{1}(\mathcal{O}(1))=\lim _{t \rightarrow \infty} \frac{t-t_{0}}{t} c_{1}(\mathcal{O}(1))=\lim _{t \rightarrow \infty} \frac{[\omega(t)]-\left[\omega\left(t_{0}\right)\right]}{t}=\lim _{t \rightarrow \infty} \frac{1}{t}[\omega(t)]
$$

We have just realized $c_{1}(\Theta(1))$ as a limit of Kähler clases. That means that the bundle $T N$ is nef by the very definition of a nef vector bundle (Definition 4.9).

We shall now apply Theorem 4.12 to Kähler quotients.

Corollary 4.13: Let $N=V / / C$ be a Kähler quotient of a vector space $V$. Suppose that $N$ is smooth and compact. If the Feix-Kaledin metric on $Y=T^{*} N$ is complete, then the tangent bundle to $N$ is big and nef.

Proof: By Theorem 3.4 the Feix-Kaledin metric is defined globally on $Y=$ $T^{*} N$. If this metric is complete, that is to say $Y=\widetilde{Y}$, then by Proposition 4.8 the moment map $\psi: Y \rightarrow \mathbb{R}_{\leq 0}$ is proper. Theorem 4.12 completes the proof of the nefness of $T N$.

By Theorem 4.2 the variety $Y_{J}=\widetilde{Y}_{J}$ is an affine algebraic variety. The result in [17], Cor. 4.4 states that this property is sufficient for $T N$ to be big (see also the remark below). 
Remark 4.14: The results in [17] are formulated for varieties called canonical extensions. These varieties are discussed in Appendix to this paper under the name twisted cotangent bundles. We believe that this name is more preferable in the context of hyperkähler geometry than the name used in [17]. In particular, Corollary 5.6 states that canonical extensions/twisted cotangent bundles are isomorphic to $Y_{J}$ (the variety $Y$ with "twisted" complex structure). That is why we can apply [17] in the proof of Corollary 4.13.

The property of a variety to have the tangent bundle which is big and nef is quite restrictive. By [24] the varieties with this property are necessarily Fano. The only known examples of such varieties are rational homogeneous. A famous conjecture by Campana-Peternell ([7]) predicts that rational homogeneous varieties exhaust the class of Fano varieties with nef tangent bundle.

\section{Appendix: Feix Construction and Twisted Cotan- gent Bundles}

Let $X$ be a compact Kähler manifold with a fixed Kähler form $\omega \in \Lambda^{1,1}(X)$. Consider the set of locally free sheaves $\mathscr{E}$ over $X$ which are extensions of the form

$$
0 \rightarrow \Omega X \rightarrow \mathcal{E} \rightarrow \mathcal{O}_{X} \rightarrow 0
$$

Their isomorphism classes are classified by $\operatorname{Ext}^{1}\left(\mathcal{O}_{X}, \Omega X\right) \cong H^{1}(X, \Omega X) \cong$ $H^{1,1}(X)$. The first isomorphism holds by general homological algebra results, the second one follows from the classical Hodge theory.

\section{Definition 5.1:}

(i) Consider the extension $\mathscr{E}$ corresponding to $[\omega] \in H^{1,1}(X)=\operatorname{Ext}^{1}\left(\mathcal{\Theta}_{X}, \Omega X\right)$. We shall call $\&$ the twistor sheaf of $X$.

(ii) Let $E$ be the vector bundle whose sheaf of sections is $\mathcal{E}$. Consider the map of vector bundles $\rho: E \rightarrow \underline{\mathbb{C}}$ induced by the surjection in the short exact sequence (5.1). Then the preimage in $E$ of the unit section of the trivial bundle $\mathbb{C}$ is an affine bundle over $X$ called the twisted cotangent bundle ([1]). We shall denote it by $\Omega X^{t w}$.

The manifold $\operatorname{Tot}(E)$ admits a smooth projection $T^{\prime}: \operatorname{Tot}(E) \rightarrow \mathbb{A}^{1}$ which we define as a composition of the map $\rho: \operatorname{Tot}(E) \rightarrow X \times \mathbb{A}^{1}$ with the projection to the second factor. Its fiber over $0 \in \mathbb{A}^{1}$ is $T^{*} X:=\operatorname{Tot}(\Omega X)$. Hence we constructed a deformation of $T^{*} X$ over $\mathbb{A}^{1}$.

We shall now rewrite the Feix construction ([11]) of the Feix-Kaledin hyperkähler structure on a neighbourhood $U$ of the zero section of $T^{*} X$ in more 
canonical terms. That will allow us to see that her construction is deeply related with twistor sheaves defined above. This relation is not transparent from her papers.

Let $X$ be a complex manifold. Then the manifold $X^{\mathbb{C}}:=X \times \bar{X}$ is the canonical complexification of the real manifold $X_{\mathbb{R}}$. Indeed, the manifold $X_{\mathbb{R}}$ admits a totally real embedding

$$
\Delta: X \rightarrow X \times \bar{X} \quad x \mapsto(x, x)
$$

Denote by $\pi: X \times \bar{X} \rightarrow X$ the projection to the first factor.

Now let $\omega \in \Lambda^{1,1}(X)$ be a real-analytic Kähler form on $X$. The form $\omega$ can be extended to the holomorphic 2 -form $\omega^{\mathbb{C}}$ on a neighbourhood $U$ of $\Delta(X)$ in $X^{\mathbb{C}}$. Let $\left(z_{1}, z_{2}, \ldots z_{n}\right)$ be holomorphic coordinates on $X$ such that

$$
\omega=\sum_{i, j} h_{i j} d z_{i} \wedge d \overline{z_{j}}
$$

where $h_{i j}$ are local real analytic function on $X$. Then $\left(z_{1}, \ldots z_{n}, w_{1}, . . w_{n}\right)$ where $w_{i}:=\overline{z_{i}}$ is a holomorphic coordinate system on $X \times \bar{X}$. The complexified form $\omega^{\mathbb{C}}$ is locally given as

$$
\omega^{\mathbb{C}}=\sum_{i, j} \widetilde{h_{i j}} d z_{i} \wedge d w_{j}
$$

where $\widetilde{h_{i j}}$ is the holomorphic extension of $h_{i j}$. We shall abuse the notation and denote the neighbourhood $U$ on which $\omega^{\mathbb{C}}$ is defined again by $X^{\mathbb{C}}$ though $U$ may be smaller.

The coordinate description allows one to see clearly that the projection $\pi: X^{\mathbb{C}} \rightarrow X$ is a holomorphic Lagrangian fibration with smooth fibers. Let $\mathscr{T}\left(X^{\mathbb{C}} / X\right)$ and $\Omega\left(X^{\mathbb{C}} / X\right)$ be the relative tangent and cotangent bundle respectively of $X^{\mathbb{C}}$ with respect to $X$. We define a holomorphic fiberwise connection $\nabla: \mathscr{T}\left(X^{\mathbb{C}} / X\right) \rightarrow \mathscr{T}\left(X^{\mathbb{C}} / X\right) \otimes \Omega\left(X^{\mathbb{C}} / X\right)$ on the fibers of the fibration $\pi$ by the following formula

$$
\left.\left.\left.\left(\nabla_{V} U\right)\right\lrcorner \omega^{\mathbb{C}}=V\right\lrcorner\left(d(U\lrcorner \omega^{\mathbb{C}}\right)\right)
$$

for every holomorphic vector fields $V, U$ tangent to the fibers. We shall call $\nabla$ the Liouville-Arnold connection. The following fact is well-known ([11]).

Proposition 5.2: Liouville-Arnold connection $\nabla: \mathcal{T}\left(X^{\mathbb{C}} / X\right) \rightarrow \mathscr{T}\left(X^{\mathbb{C}} / X\right) \otimes$ $\Omega\left(X^{\mathbb{C}} / X\right)$ is well-defined by formula (5.3). Furthermore, it is flat and torsionfree along the fibers.

Let $\mathscr{F}_{0}$ be a subsheaf of $\pi_{*} \Omega\left(X^{\mathbb{C}} / X\right)$ consisting of 1 -forms parallel with respect to $\nabla$. The flatness of $\nabla$ implies that $\mathscr{F}_{0}$ is locally free.

Proposition 5.3: The locally free sheaf $\mathscr{F}_{0}$ on $X$ is naturally isomorphic to $\mathcal{T} X$. 
Proof: A 1-form $\alpha \in \Omega\left(X^{\mathbb{C}} / X\right)$ is parallel if and only if for every vector fields $V, U$ tangent to the fibers

$$
\left.\left.\left(\nabla_{V} U\right)\right\lrcorner \alpha=V(U\lrcorner \alpha\right)
$$

Let $\chi$ be a holomorphic vector field on $X$. It defines a vector filed $\chi_{0}:=(\chi, 0)$ on $\pi^{-1}(U) \subset X^{\mathbb{C}} \subset X \times \bar{X}$. Then the form $\left.\alpha:=\chi_{0}\right\lrcorner \omega^{\mathbb{C}}$ is $\nabla$-parallel. Indeed,

$$
\begin{gathered}
\left.\left.\left(\nabla_{V} U\right)\right\lrcorner \alpha=-\omega^{\mathbb{C}}\left(\nabla_{V} U, \chi_{0}\right)=d(U\lrcorner \omega^{\mathbb{C}}\right)\left(\chi_{0}, V\right)= \\
=\chi_{0}\left(\omega^{\mathbb{C}}(U, V)\right)-V\left(\omega^{\mathbb{C}}\left(U, \chi_{0}\right)-\omega^{\mathbb{C}}\left(U,\left[\chi_{0}, V\right]\right)\right.
\end{gathered}
$$

The second equality follows from the definition of $\nabla$ and the third one from the Cartan formula for the de Rham differential. The term $\chi_{0}\left(\omega^{\mathbb{C}}(U, V)\right)$ vanishes as the fibers are Lagrangian. The term $\omega^{\mathbb{C}}\left(U,\left[\chi_{0}, V\right]\right)$ vanishes for the same reason because $\left[\chi_{0}, V\right]$ is tangent to the fibers. Hence

$$
\left.\left.\left(\nabla_{V} U\right)\right\lrcorner \alpha=V\left(\omega^{\mathbb{C}}\left(\chi_{0}, U\right)\right)=V(U\lrcorner \alpha\right)
$$

and $\alpha$ is indeed parallel.

The map of locally free sheaves $\mathcal{T} X \rightarrow \mathscr{F}_{0}$ sending $\chi$ to $\alpha$ is injective as the 2 -form $\omega^{\mathbb{C}}$ is non-degenerate. The equality of the ranks of $\mathcal{T} X$ and $\mathscr{F}_{0}$ implies that this map is an isomorphism.

The vanishing of the torsion of $\nabla$ implies that every parallel form is closed. Shrinking $X^{\mathbb{C}}$ if necessary we may assume that the fibers of $\pi: X^{\mathbb{C}} \rightarrow X$ are simply connected. Consider the map of sheaves of $\mathcal{O}_{X}$-modules

$$
d: \pi_{*} \mathcal{O}_{X^{\mathbb{C}} / X} \rightarrow \Omega\left(X^{\mathbb{C}} / X\right)
$$

where $d$ is the de Rham differential. Notice that it is indeed a map of sheaves of $\mathcal{O}_{X}$-modules. We define $\mathscr{F} \subset \pi_{*} \mathcal{O}_{X^{\mathbb{C}} / X}$ to be the preimage of $\mathscr{F}_{0} \subset \Omega\left(X^{\mathbb{C}} / X\right)$ by the map $d$.

By the assumption of simply-connectedness of the fibers the map $d: \mathscr{F} \rightarrow$ $\mathscr{F}_{0} \cong \mathfrak{T} X$ is surjective with the kernel $\mathcal{O}_{X}$. We obtain an extension

$$
0 \rightarrow \mathcal{O}_{X} \rightarrow \mathcal{F} \rightarrow \mathcal{T} X \rightarrow 0
$$

Remark 5.4: This extension is not in general non-trivial as an extension of locally free sheaves of $\mathcal{O}_{X}$-modules. Nevertheless, there exists a real splitting of the short exact sequence (5.5). We have a $C^{\infty}(X)$-linear map

$$
e v_{\Delta}: C^{\infty}(\mathcal{F}) \rightarrow C^{\infty}(X) \quad e v_{\Delta}(f):=\left.f\right|_{\Delta(X)}
$$

which is the desired splitting. 
It will be more convenient for us to work with the dualiziation of the short exact sequence (5.5)

$$
0 \rightarrow \Omega X \rightarrow \mathscr{F}^{\vee} \rightarrow \mathcal{O}_{X} \rightarrow 0
$$

Theorem 5.5: The sheaf $\mathcal{F}^{\vee}$ is the twistor sheaf of $X$ (Definition 5.1).

Proof. Step 1: The cohomology of the sheaf $\Omega X$ can be computed by two different complexes. The first one is the $\bar{\partial}$-complex

$$
\Lambda^{1, \bullet}(X)=\left\{0 \rightarrow \Lambda^{1,0}(X) \rightarrow \Lambda^{1,1}(X) \rightarrow \Lambda^{1,2}(X) \rightarrow \ldots\right\}
$$

Now let us choose a locally finite covering of $X$ by affine charts so that $X=\bigcup_{i} U_{i}$. Then the Čech complex $\check{C} \bullet(\Omega X)$ assosiated with this covering also computes the cohomology of $\Omega X$. This complex is given explicitly as

$$
\check{C} \bullet(\Omega X)=\left\{0 \rightarrow \bigoplus_{i} \Omega X\left(U_{i}\right) \rightarrow \bigoplus_{i<j} \Omega X\left(U_{i j}\right) \rightarrow \bigoplus_{i<j<k} \Omega X\left(U_{i j k}\right) \rightarrow \ldots\right\}
$$

where for any subset $I$ of indices we denote $\bigcap_{i \in I} U_{i}$ by $U_{I}$.

Let $[\omega] \in H^{1}\left(\Lambda^{1, \bullet}(X)\right)$ be a cohomology class represented by the closed Kähler form $\omega \in \Lambda^{1,1}(X)$. Let $\omega_{i}$ be the restriction of $\omega$ to $U_{i}$. On every open subset $U_{i}$ choose a Kähler potential $f_{i}$ i.e. the function $f_{i}: U_{i} \rightarrow \mathbb{R}$ such that

$$
\sqrt{-1} \partial \bar{\partial} f_{i}=\omega_{i}
$$

For every pair of indices $i<j$ consider the holomorphic form $\eta_{i j}:=\sqrt{-1} \partial\left(\left.f_{i}\right|_{U_{i j}}-\right.$ $\left.\left.f_{j}\right|_{U_{i j}}\right) \in \Omega X\left(U_{i j}\right)$. We claim that the element $\left(\eta_{i j}\right) \in \bigoplus_{i<j} \Omega X\left(U_{i j}\right)$ represents the same cohomology class in $H^{1}(X, \Omega X)$ as $[\omega]$. That follows from standard homological algebra arguments. More precisely, one first identifies the cohomology groups of $\Lambda^{1, \bullet}(X)$ and $\check{C} \bullet(\Omega X)$ with the cohomology groups of the total complex of the following bicomplex

$$
A^{p, q}=\bigoplus_{|I|=q} \Omega^{1, p}\left(U_{I}\right)
$$

and then use a simple diagram chasing. Details can be found for example in Stacks project ([25] Part 1, Ch. 12, Section 12.25).

Step 2: As the Kähler form $\omega$ is real analytic, a local Kähler potential $f_{i}: U_{i} \rightarrow \mathbb{R}$ may also be chosen to be real analytic. Let $\widetilde{f}_{i}$ be its holomorphic extension to $U_{i}^{\mathbb{C}} \subset U_{i} \times \overline{U_{i}}$. Then

$$
\left.\omega^{\mathbb{C}}\right|_{U_{i}^{\mathbb{C}}}=\sqrt{-1} d_{0} d_{1} \widetilde{f}_{i}
$$

where $d_{0}$ is the component of $d$ in the direction of $U_{i}$ and $d_{1}$ is the component of $d$ in the direction of $\overline{U_{i}}$. By the proof of Proposition 5.3 the map sending 
a holomorphic vector field $\chi$ on $X$ to $\left.\chi_{0}\right\lrcorner \omega^{\mathbb{C}}$ induces an isomorphism between the tangent bundle $\mathcal{T} X$ and the bundle $\mathscr{F}_{0}$ of parallel 1-forms on the fibers of $\pi: X^{\mathbb{C}} \rightarrow X$. Locally

$$
\begin{gathered}
\left.\left.\left.\chi_{0}\right\lrcorner \omega^{\mathbb{C}}=\sqrt{-1} \chi_{0}\right\lrcorner d_{0} d_{1} \widetilde{f}_{i}=\sqrt{-1} \chi_{0}\right\lrcorner d d_{1} \widetilde{f}_{i}= \\
=\sqrt{-1} \mathrm{~L}_{\chi_{0}} d_{1} \widetilde{f}_{i}=\sqrt{-1} d_{1}\left(\chi_{0}\left(\widetilde{f}_{i}\right)\right)=d_{1}\left(\sqrt{-1} \widetilde{\chi\left(f_{i}\right)}\right)
\end{gathered}
$$

We thus obtain a local holomorphic splitting $\iota_{i}: \mathfrak{T} X \rightarrow \mathscr{F}$ of the short exact sequence (5.5)

$$
\iota_{i}(\chi):=\sqrt{-1} \widetilde{\chi\left(f_{i}\right)}
$$

Step 3: The short exact sequence (5.7) induces the long exact sequence of cohomology groups

$$
0 \rightarrow H^{0}(X, \Omega X) \rightarrow H^{0}\left(X, \mathcal{F}^{\vee}\right) \rightarrow H^{0}\left(X, \mathcal{O}_{X}\right) \rightarrow H^{1}(X, \Omega X) \rightarrow \ldots
$$

By a standard result from homological algebra the class of the extension $\mathcal{F}^{\vee}$ in $H^{1}(X, \Omega X)$ is the image of the unit section $\mathbb{1}_{X} \in H^{0}\left(X, \mathcal{O}_{X}\right)$ in $H^{1}(X, \Omega X)$. We compute it in terms of the Čech cohomology. One has a complex of short exact sequences

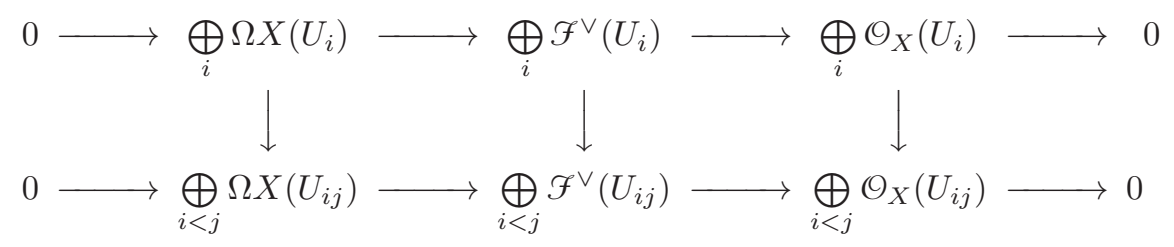

The morphism $H^{0}\left(X, \mathcal{O}_{X}\right) \rightarrow H^{1}(X, \Omega X)$ is given explicitly as follows. In Step 2 we constructed a local splitting of the short exact sequence (5.7). Hence we have a lift of the element $\left(\left.\mathbb{1}\right|_{U_{i}}\right) \in \bigoplus_{i} \mathcal{O}\left(U_{i}\right)$ to an element of $\bigoplus_{i} \mathcal{F}^{\vee}\left(U_{i}\right)$. We shall denote its component in $\mathcal{F}^{\vee}\left(U_{i}\right)$ by $\mathbb{1}_{i}$. It is uniquely defined by the properties

$$
\mathbb{1}_{i}(h)=h
$$

for every holomorphic function $h$ on $X$, and

$$
\mathbb{1}_{i}\left(\widetilde{\chi\left(f_{i}\right)}\right)=0
$$

for any holomorphic every vector field $\chi$ on $X$.

The downward pointing differential sends $\left(\mathbb{1}_{i}\right) \in \bigoplus_{i} \mathcal{F}^{\vee}\left(U_{i}\right)$ to $\left(\left.\mathbb{1}_{j}\right|_{U_{i j}}-\right.$ $\left.\left.\mathbb{1}_{i}\right|_{U_{i j}}\right) \in \bigoplus_{i<j} \mathcal{F}^{\vee}\left(U_{i j}\right)$. This last element in fact lies in the image of $\bigoplus_{i} \Omega X\left(U_{i}\right)$. Let $\left(\eta_{i j}^{\prime}\right) \in \bigoplus_{i} \Omega X\left(U_{i}\right)$ be its preimage. Then for every local vector field $\chi$ on $X$

$$
\begin{aligned}
\eta_{i j}^{\prime}(\chi)= & \left(\left.\mathbb{1}_{j}\right|_{U_{i j}}-\left.\mathbb{1}_{i}\right|_{U_{i j}}\right)\left(\iota_{i}(\chi)\right)=\sqrt{-1}\left(\left.\mathbb{1}_{j}\right|_{U_{i j}}-\left.\mathbb{1}_{i}\right|_{U_{i j}}\right)\left(\widetilde{\chi\left(f_{i}\right)}\right)= \\
= & \left.\sqrt{-1} \mathbb{1}_{j}\right|_{U_{i j}}\left(\widetilde{\chi\left(f_{i}\right)}-\widetilde{\chi\left(f_{j}\right)}\right)=\sqrt{-1} \chi\left(f_{i}-f_{j}\right)
\end{aligned}
$$


where the last identity holds as the function $\chi\left(f_{i}\right)-\chi\left(f_{j}\right)$ is a holomorphic function on $X$. We now readily see that $\eta_{i j}^{\prime}=\sqrt{-1} \partial\left(f_{i}-f_{j}\right)$. By the results of Step 1 of the proof $\eta_{i j}^{\prime}=\eta_{i j}$. Consequently, the element $\left(\eta_{i j}^{\prime}\right) \in \bigoplus_{i} \Omega X\left(U_{i}\right)$ represents the same cohomology class in $H^{1}(X, \Omega X)$ as the Kähler form $\omega$. By Definition 5.1 the locally free sheaf $\mathcal{F}^{\vee}$ is the twistor sheaf.

Corollary 5.6: For a small enough neighbourhood $U$ of the zero section of $T^{*} M$ there exists a hyperkähler metric on $U$ with the following property. Let $T: \operatorname{Tw}(U) \rightarrow \mathbb{P}^{1}$ be the twistor deformation. Let $\mathcal{E}$ be the twistor sheaf of $X$ and $E$ be the corresponding vector bundle. Then their exist a holomorphic open embedding $u: T^{-1}\left(\mathbb{A}^{1}\right) \rightarrow \operatorname{Tot}(E)$ commuting with the projection to $\mathbb{A}^{1}$. In other words, the following diagram commutes.

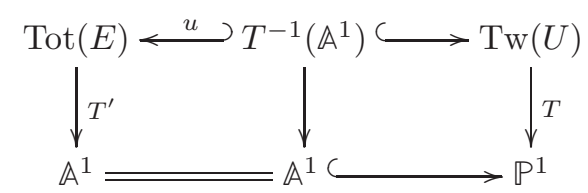

Proof: Feix in [11] constructs a complex manifold $Z$ with a projection $T: Z \rightarrow$ $\mathbb{P}^{1}$ which turns out to be the twistor projection for some hyperkähler metric on $U$. She obtains $Z$ by gluing the complex manifolds $\operatorname{Tot}\left(F^{*}\right)$ and $\operatorname{Tot}\left(\overline{F^{*}}\right)$ over $\mathbb{P}^{1}$ by means of a certain holomorphic map. In particular, by the very construction of her the manifold $\operatorname{Tot}\left(F^{*}\right)$ is isomorphic to $T^{-1}\left(\mathbb{A}^{1}\right)$. The statement of the corollary now follows from Theorem 5.5.

\section{References}

[1] Beilinson, Alexander A., and David Kazhdan. "Flat projective connections." unpublished manuscript, https://www.math.stonybrook.edu/ kirillov/manuscripts.html. (Cited on page 32.)

[2] Bielawski, Roger. "Ricci-flat Kähler metrics on canonical bundles." Math. Proc. Cambridge Philos. Soc. 132, no. 3 (2002): 471-479, arXiv:math/0006144. (Cited on page 31.)

[3] Bielawski, Roger. "Complexification and hypercomplexification of manifolds with a linear connection." Internat. J. Math. 14, no. 8 (2003): 813-824, arXiv:math/0212175. (Cited on page 3.)

[4] Bielawski, Roger, and Andrew S. Dancer. "The geometry and topology of toric hyperkähler manifolds." Comm. Anal. Geom. 8, no. 4 (2000): 727-759. (Cited on pages 2 and 20.)

[5] Biquard, Olivier, and Paul Gauduchon. "Géométrie hyperkählérienne des espaces hermitiens symétriques complexifiés." In Séminaire de théorie spectrale et géométrie 16 (1997-1998), 127-173. (Cited on page 2.) 
[6] Borówka, Aleksandra W., and David M.J. Calderbank. "Projective geometry and the quaternionic Feix-Kaledin construction." Trans. Amer. Math. Soc. 372, no.7 (2019): 4729-4760, arXiv:1512.07625. (Cited on page 3.)

[7] Campana, Frédéric, and Thomas Peternell. "Projective manifolds whose tangent bundles are numerically effective." Math. Ann. 289, no. 1 (1991): 169188. (Cited on page 32.)

[8] Cannas da Silva, Ana. Lectures on symplectic geometry. Lecture Notes in Mathematics 1764, Springer-Verlag, 2008. (Cited on page 26.)

[9] Dancer, Andrew S., and Andrew F. Swann. "The geometry of singular quaternionic Kähler quotients." Internat. J. Math. 8, no. 5 (1997): 595610. (Cited on page 7.)

[10] Demailly, Jean-Pierre. Complex analytic and differential geometry. open content book, https://www-fourier.ujf-grenoble.fr/ demailly/documents.html. (Cited on page 26.)

[11] Feix, Birte. "Hyperkähler metrics on cotangent bundles." J. Reine Angew. Math. 532 (2001): 33-46. (Cited on pages 2, 32, 33, and 37.)

[12] Feix, Birte. "Twistor spaces of hyperkähler manifolds with $S^{1}$-actions." Differential Geom. Appl. 19, no.1 (2003): 15-28. (Cited on page 2.)

[13] Fornæss, John E., and Raghavan Narasimhan. "The Levi problem on complex spaces with singularities." Math. Ann. 248 (1980): 47-72. (Cited on pages 4 and 26.)

[14] Georgoulas, Valentina, Joel W. Robbin, and Dietmar A. Salamon. "The moment-weight inequality and the Hilbert-Mumford criterion." arXiv preprint (2013), arXiv:1311.0410. (Cited on pages 11 and 12.)

[15] Greb, Daniel. "Compact Kähler quotients of algebraic varieties and geometric Invariant Theory." Adv. Math. 224, no.2 (2010): 401-431, arXiv:0812.2643. (Cited on page 7.)

[16] Greb, Daniel, and Christian Miebach. "Invariant meromorphic functions on Stein spaces." Ann. Inst. Fourier (Grenoble) 62, no.5 (2012): 1983-2011, arXiv:1010.2935. (Cited on page 8.)

[17] Greb, Daniel, and Michael L. Wong. "Canonical complex extensions of Kähler manifolds." J. London Math. Soc. 101 no. 2 (2020): 786-827, arXiv:1807.01223. (Cited on pages 31 and 32.)

[18] Hartshorne, Robin. Ample Subvarieties of Algebraic Varieties. Lecture Notes in Mathematics 156, Springer-Verlag, 1970. (Cited on page 26.)

[19] Haydys, Andriy. "HyperKähler and quaternionic Kähler manifolds with $S^{1}$ symmetries." J. Geom. Phys. 58, no.3 (2008): 293-306. (Cited on page 2.) 
[20] Heinzner, Peter, and Alan Huckleberry. "Analytic Hilbert quotients." In Several Complex Variables edited by M. Schneider, Y.-T. Siu, 309-349. Mathematical Sciences Research Institute Publications 37, Cambridge University Press, 1999. (Cited on pages 8, 11, and 12.)

[21] Hitchin, Nigel J., Anders Karlhede, Ulf Lindström, and Martin Roček. "Hyperkähler metrics and supersymmetry." Comm. Math. Phys. 108, no.4 (1987): 535-589. (Cited on pages 3, 5, 26, and 27.)

[22] Hoskins, Victoria. "Stratifications associated to reductive group actions on affine spaces." Q. J. Math. 65, no.3 (2014): 1011-1047, arXiv:1210.6811. (Cited on page 15.)

[23] Hoskins, Victoria. "Moduli problems and geometric invariant theory." lecture notes, 2015-2016, https://userpage.fuberlin.de/hoskins/M15_Lecture_notes.pdf. (Cited on pages 7 and 8.)

[24] Hsiao, Jen-Chieh. "A remark on bigness of the tangent bundle of a smooth projective variety and D-simplicity of its section rings." J. Algebra Appl. 14, no.7 (2015) 1550098. (Cited on page 32.)

[25] de Jong, A. J. et al., Stacks project, collaborative textbook project, https://stacks.math.columbia.edu/. (Cited on page 35.)

[26] Kaledin, Dmitry. "Singular hyperkähler quotients." PhD diss., Massachusetts Institute of Technology, 1995. (Cited on page 7.)

[27] Kaledin, Dmitry. "Hyperkähler structures on total spaces of holomorphic cotangent bundles." In D. Kaledin, M. Verbitsky, New constructions of hyperkähler manifolds, Intl. Press, Cambridge, MA, 2000, arXiv:math/0011256. (Cited on page 2.)

[28] Kaledin, Dmitry. "A canonical hyperkähler metric on the total space of a cotangent bundle." In Proceeedings of the Second Quaternionic Meeting. Rome, World Scientific, Singapore, 1999, arXiv:math/0011256. (Cited on page 2.)

[29] Kovalev, Alexei G. "Nahm's equations and complex adjoint orbits." Q. J. Math. 47, no.1 (1996): 41-58. (Cited on page 2.)

[30] Luna, Domingo. "Fonctions différentiables invariantes sous l'opération d'un groupe réductif." Ann. Inst. Fourier (Grenoble) 26, no.1 (1976): 33-49. (Cited on page 8.)

[31] Mayrand, Maxence. "Stratified hyperkähler spaces and Nahm's equations." PhD diss., Oxford University, 2019. (Cited on pages 1, 7, and 20.)

[32] Mumford, David B., John Ch. Fogarty, and Frances C. Kirwan. Geometric invariant theory. Ergebnisse der Mathematik und ihrer Grenzgebiete (2) [Results in Mathematics and Related Areas (2)], 34 (3rd ed.), Berlin, New York: Springer-Verlag, 1994. (Cited on page 8.) 
[33] Mundet i Riera, Ignasi. "A Hitchin-Kobayashi correspondence for Kaehler fibrations." J. Reine Angew. Math. 528 (2000): 41-80. (Cited on page 13.)

[34] Mundet I Riera, Ignasi. "A Hilbert-Mumford criterion for polystability in Kähler geometry." Trans. Amer. Math. Soc. 362, no.10 (2010): 5169-5187. (Cited on page 13.)

[35] Nakajima, Hiraku, and Yuuya Takayama. "Cherkis bow varieties and Coulomb branches of quiver gauge theories of affine type A." Selecta Math. (N.S.) 23, no.4 (2017): 2553-2633, arXiv:1606.02002. (Cited on page 2.)

[36] Pedersen, Henrik, and Yat Sun Poon. "Hamiltonian constructions of Kähler-Einstein metrics and Kähler metrics of constant scalar curvature." Comm. Math. Phys. 136, no.2 (1991): 309-326. (Cited on page 30.)

[37] Sjamaar, Reyer. "Convexity properties of the moment mapping reexamined." Adv. Math. 138, no.1 (1998): 46-91, arXiv:dg-ga/9408001. (Cited on page 27.)

[38] Sjamaar, Reyer, and Eugene Lerman. "Stratified symplectic spaces and reduction." Ann. of Math. 134, no.2 (1991): 375-422. (Cited on page 6.)

[39] Teleman, Andrei. "Symplectic stability, analytic stability in non-algebraic complex geometry." Internat. J. Math. 15, no.2 (2004): 183-209, arXiv:math/0309230. (Cited on page 13.)

AnNa ABASheva

Laboratory of Algebraic Geometry, HSE University

Department of Mathematics, Usacheva ul., 6, Moscow, Russia

AND:

Columbia University, Department of Mathematics, 2990 Broadway, New York, NY 10027

ALSO:

INDEPENDENT UNIVERSITY OF MOSCOW

Bolshoy Vlasievskiy Per., 11, Moscow, Russia

anabasheva (at) yandex.ru. 